\title{
Impact of Diabetic Ketoacidosis on Thyroid Function in Patients with Diabetes Mellitus
}

\author{
Yuling Xing $\mathbb{D}^{1,2}$ Jinhu Chen $\mathbb{D}^{1},{ }^{1}$ Guangyao Song $\mathbb{D}^{1,3,4}$ Liying Zhao $\mathbb{D}^{1,2}$ \\ and Huijuan $\mathrm{Ma}$ iD $1,3,4$ \\ ${ }^{1}$ Department of Endocrinology, Hebei General Hospital, Shijiazhuang 050017, China \\ ${ }^{2}$ Graduate School of Hebei Medical University, Shijiazhuang 050017, China \\ ${ }^{3}$ Hebei Key Laboratory of Metabolic Diseases, Hebei General Hospital, Shijiazhuang, Hebei 050051, China \\ ${ }^{4}$ Department of Internal Medicine, Hebei Medical University, Shijiazhuang, Hebei 050017, China
}

Correspondence should be addressed to Huijuan Ma; huijuanma19@163.com

Received 11 May 2020; Revised 21 October 2020; Accepted 9 March 2021; Published 22 March 2021

Academic Editor: Ma gorzata Kotula Balak

Copyright (๑) 2021 Yuling Xing et al. This is an open access article distributed under the Creative Commons Attribution License, which permits unrestricted use, distribution, and reproduction in any medium, provided the original work is properly cited.

Background. Changes in thyroid function in diabetes patients who developed diabetic ketoacidosis (DKA) still need to be fully elucidated. The aim of this study was to systematically review available data on the relationship between thyroid function and DKA in diabetes patients who developed DKA. Methods. Electronic databases (PubMed, EMBASE, Cochrane Library, and China Academic Journal Full-text Database (CNKI)) were searched systematically to search relevant literature before December 2020. The mean\pm standard deviation and $95 \%$ confidence interval (95\% CI) were used for evaluation, and sensitivity analysis was performed. Publication bias was estimated by funnel plot, Egger's test, and Begger's test. Results. 29 studies were included in the meta-analysis, and the indicators (T4, T3, FT3, FT4, TSH, T3RU, and rT3) of patients with DKA were compared and analyzed. The results of this study showed that the levels of T4, T3, FT3, FT4, and TSH were decreased and the level of rT3 was increased in patients with DKA. Compared with after treatment, the levels of T4, T3, FT3, and FT4 in patients with DKA were decreased before treatment, while the levels of rT3 were increased, and there was no significant difference in changes of TSH. With the aggravation of DKA, the levels of T4, T3, FT3, and FT4 will further decrease, while the changes of TSH have no statistical difference. Conclusion. Thyroid function changed in diabetic patients with DKA. It changed with the severity of DKA. This condition may be transient, preceding further recovery of DKA.

\section{Introduction}

Diabetic ketoacidosis (DKA) is an acute life-threatening complication of diabetes. It is not only a sign of acute absolute insulin deficiency in type 1 diabetes mellitus (T1DM) but also increasingly seen in patients with type 2 diabetes mellitus. In patients with diabetes, ketoacidosis is caused by an acute decrease in insulin secretion and action in a severe insulin resistant state [1]. From 2002 to 2010 in the United States, about $30 \%$ of adolescents newly diagnosed with T1DM developed DKA [2]. The prevalence of DKA estimated at the onset of type 2 diabetes is quite different. African-American youth in Cincinnati and Arkansas was $41.4 \%$ [3] and $16 \%$ [4]. Statistics showed that thyroid dysfunction in people with diabetes is 2-3 times higher than people without diabetes [5]. The effect of nonthyroid diseases on thyroid function has been studied in anorexia nervosa, liver disease, kidney disease, and many other diseases [6]. Since the 1970s, it has been reported that acute disease can cause a variety of changes in the levels of thyroid hormones in patients who were not previously diagnosed with intrinsic thyroid disease. These changes are nonspecific and are related to the severity of the disease [7]. Diabetes can have a definite effect on thyroid function in various ways, leading to changes in the levels of thyroid hormones, including immunological mechanisms, cytokine pathways, and regulatory pathways of the hypothalamic-pituitarythyroid axis [8]. When DKA occurred in patients with diabetes, the changes in thyroid function has received a great deal of attention from researchers. At present, there are 
limited studies on the changes in levels of thyroid hormone in patients with DKA. DKA and its implication in the thyroid function has not been adequately reviewed. The study aimed to analyze the changes in the levels of thyroid hormones in patients with DKA and the relationship between the changes and the severity of DKA.

\section{Materials and Methods}

2.1. Literature Search Strategy. Diabetic ketoacidosis, related indicators reflecting thyroid function (free triiodothyronine (FT3), free thyroxine (FT4), triiodothyronine (T3), thyroxine (T4), thyroid-stimulating hormone (TSH), T3 resin uptake (T3RU), and reverse triiodothyronine (rT3)) as subject terms and keywords for joint search. All relevant literature published before December 2020 was searched in PubMed, EMBASE, Cochrane Library, and CNKI.

2.2. Inclusion Criteria. (1) The article related to patients with DKA; (2) involving the changes of thyroid function indicators in patients with DKA before and after treatment or between the diabetic patients with and without DKA and providing the exact sample size and data on various indicators of thyroid function; and (3) the diagnosis of diabetic ketoacidosis is clear [9].

2.3. Exclusion Criteria. (1) The data of literature are incomplete and the information is not enough to calculate the statistics of this study; (2) case reports; (3) repeated articles; and (4) studies limited to animals.

2.4. Literature Screening. Two researchers independently screened the literature, extracted data, and cross-checked. If there is a disagreement on the results, they would discuss it together or resolve it by a third senior researcher. In the study, data were extracted from the literature finally included in the meta-analysis using a premade data extraction table. The extracted content included the first author, year of publication, study area, sample size, mean \pm standard deviation of thyroid function indicators, inclusion criteria, exclusion criteria, DKA diagnostic cutoff point, the determination method of thyroid hormone, therapeutic approach, and duration of treatment of DKA (Table 1).

2.5. Statistical Analysis. According to the requirements of meta-analysis, the data were sorted out, the database was established, the data were carefully checked, and the standardized mean difference (SMD) and 95\% CI were used to quantitatively analyze the measurement data. I2 was used to quantitatively test the heterogeneity among the studies. If I $2 \leq 50 \%$, it was considered that the heterogeneity was not statistically significant, and the fixed effect model was used to analyze; on the contrary, if I $2>50 \%$, the heterogeneity was considered to be statistically significant, and the random effect model was used to analyze. Sensitivity analysis was performed to ensure the stability of the meta-analysis results. Funnel plot and Egger's test were used to evaluate publication bias, and $p<0.05$ was considered as statistically significant, indicating that publication bias was not excluded. The trim-and-fill method was used to estimate the effect of publication bias on the interpretation of the results.

\section{Result}

3.1. Literature Search Results. 314 related studies were initially retrieved based on keywords and subject terms, and finally, 29 studies met the predetermined inclusion and exclusion criteria (Figure 1). 17 studies evaluated the changes of thyroid function before and after treatment in patients with DKA, 17 studies evaluated the difference of thyroid function between patients with diabetes with and without DKA, and 3 studies related to the changes of thyroid function with different severities of DKA. The relevant literature was published from 1978 to 2018 (Tables 1-3).

\subsection{Meta-Analysis Results}

3.2.1. Comparison of Thyroid Function between Patients with Diabetes with and without DKA. 15 studies involved the comparison of T4 between patients with diabetes with and without DKA, involving 751 patients with DKA and 817 patients with diabetes but without DKA; 16 studies involved the comparison of T3, involving 755 patients with DKA and 828 patients with diabetes but without DKA; 15 studies involved the comparison of FT4, involving 790 patients with DKA and 876 patients with diabetes but without DKA; 12 studies involved the comparison of FT3, involving 643 patients with DKA and 744 patients with diabetes but without DKA; 16 studies involved the comparison of TSH, involving 848 patients with diabetes and DKA and 981 patients with diabetes but without DKA; and 6 studies involved the comparison of rT3, involving 135 patients with DKA and 194 patients with diabetes but without DKA. The results showed that compared with patients with diabetes, patients with DKA had lower levels of T4, T3, FT4, and FT3 and higher level of rT3. The difference was statistically significant (T4:I2 =83.9\%, $p<0.01, \quad Z=7.2, \quad p<0.01$, $\mathrm{SMD}=-1.030,95 \% \mathrm{CI}:-1.310$ to -0.749 ; T3 : $\mathrm{I} 2=82.1 \%$, $p<0.01, Z=7.4, p<0.01, \mathrm{SMD}=-1.022,95 \% \mathrm{CI}:-1.292$ to $-0.751 ; \quad \mathrm{FT} 4: \mathrm{I} 2=93.9 \%, \quad p<0.01, \quad Z=3.45, \quad p<0.01$, $\mathrm{SMD}=-0.758,95 \% \mathrm{CI}:-1.189$ to -0.327 ; FT3 $: \mathrm{I} 2=89.6 \%$, $p<0.01, Z=4.82, p<0.01, \mathrm{SMD}=-0.884,95 \% \mathrm{CI}:-1.243$ to $-0.524 ; \quad$ rT3 : I2 $=95.8 \%, \quad p<0.01, \quad Z=3.15, \quad p<0.01$, $\mathrm{SMD}=2.534,95 \% \mathrm{CI}: 0.956$ to 4.112 ; $\mathrm{TSH}: \mathrm{I} 2=61.1 \%$, $p<0.01, Z=1.33, p=0.185, \mathrm{SMD}=-0.106,95 \% \mathrm{CI}:-0.261$ to 0.05 ; Figure 2). There was no statistical difference in TSH between patients with diabetes with and without DKA. After sensitivity analysis, the result showed that TSH was significantly different ( $\mathrm{I} 2=42.6 \%, p<0.05, Z=2.01, p<0.05$, $\mathrm{SMD}=-0.138,95 \% \mathrm{CI}:-0.273$ to -0.003 Figure 3$)$. Therefore, patients with DKA have lower levels of T4, T3, FT4, FT3, and TSH and higher level of rT3. Egger's test (T4, $p=0.861$; FT4, $p=0.504$; rT3, $p=0.445$ ) showed that there was no obvious publication bias. Further analysis by the cutand-fill method showed that the publication bias (T3, $p=0.043$; FT3, $p=0.003$; TSH, 0.003) did not affect the 


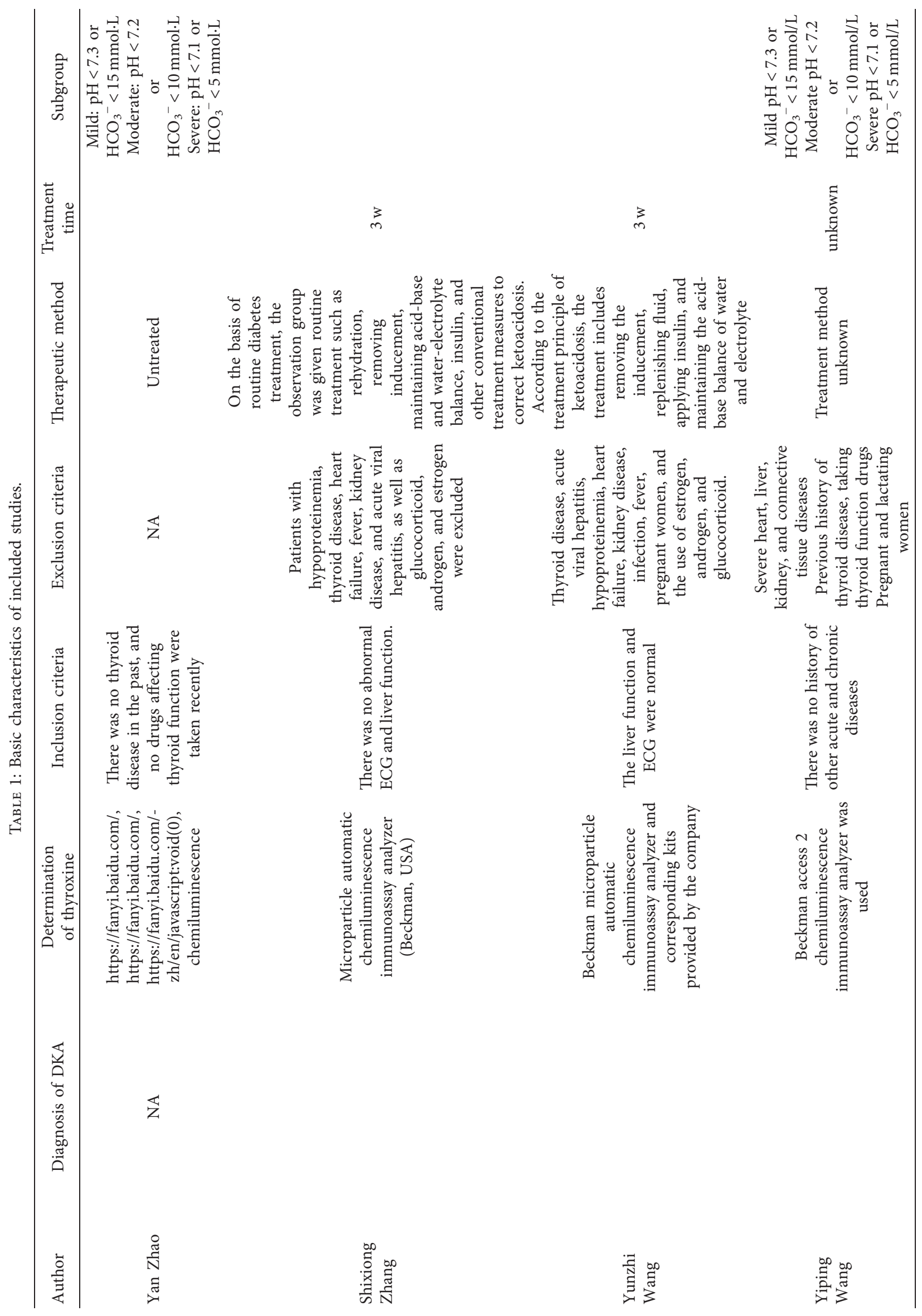




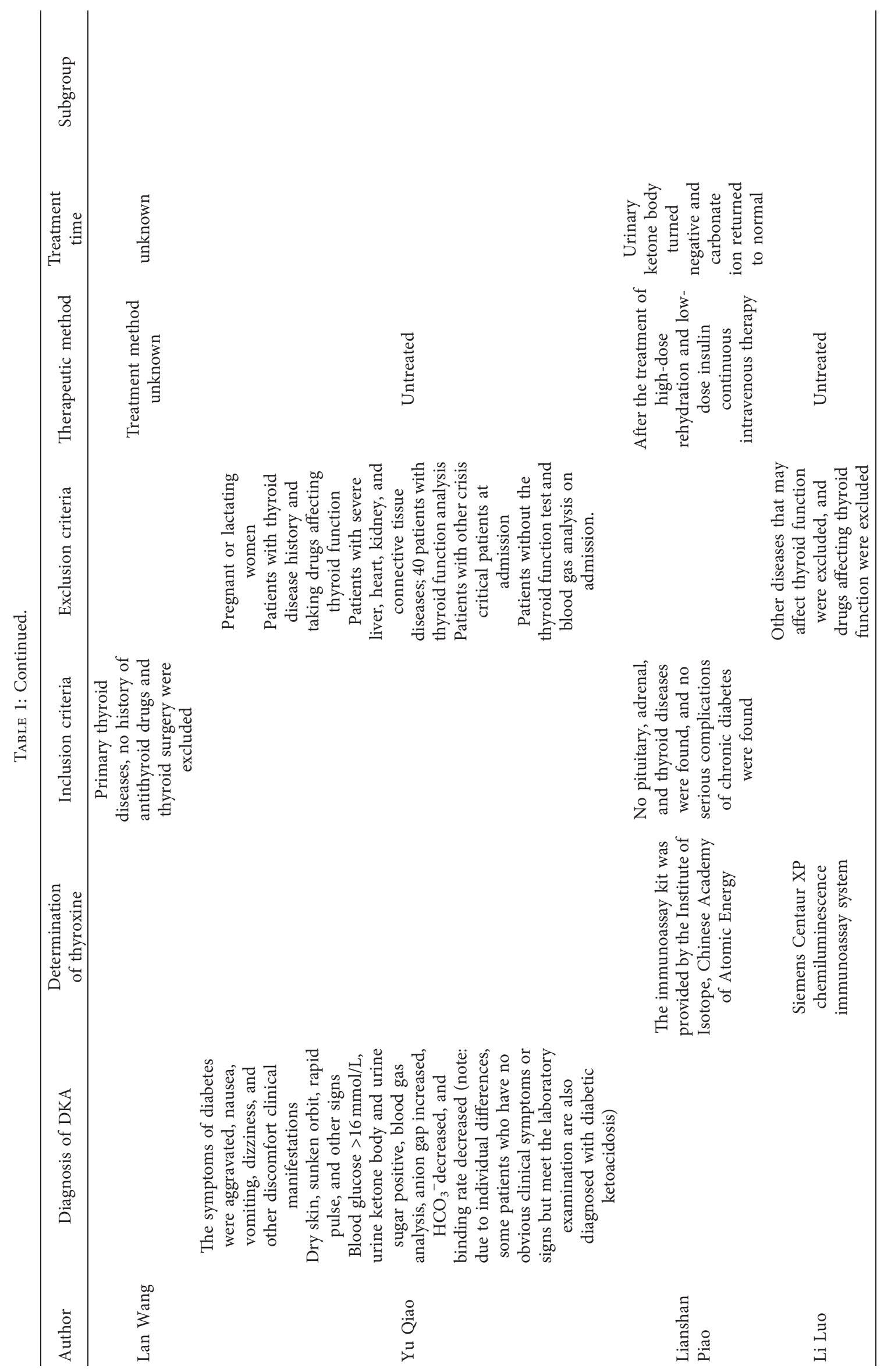




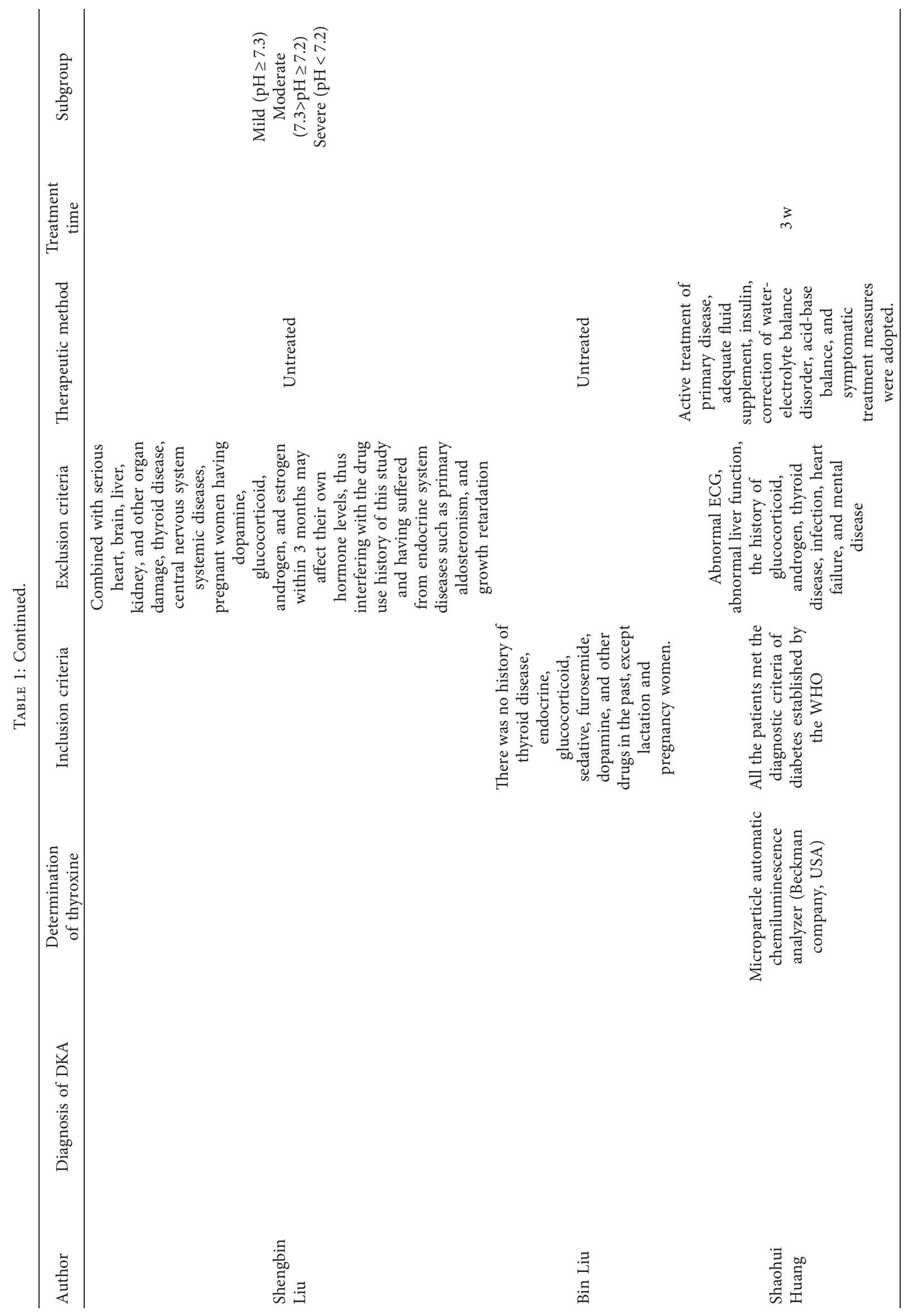




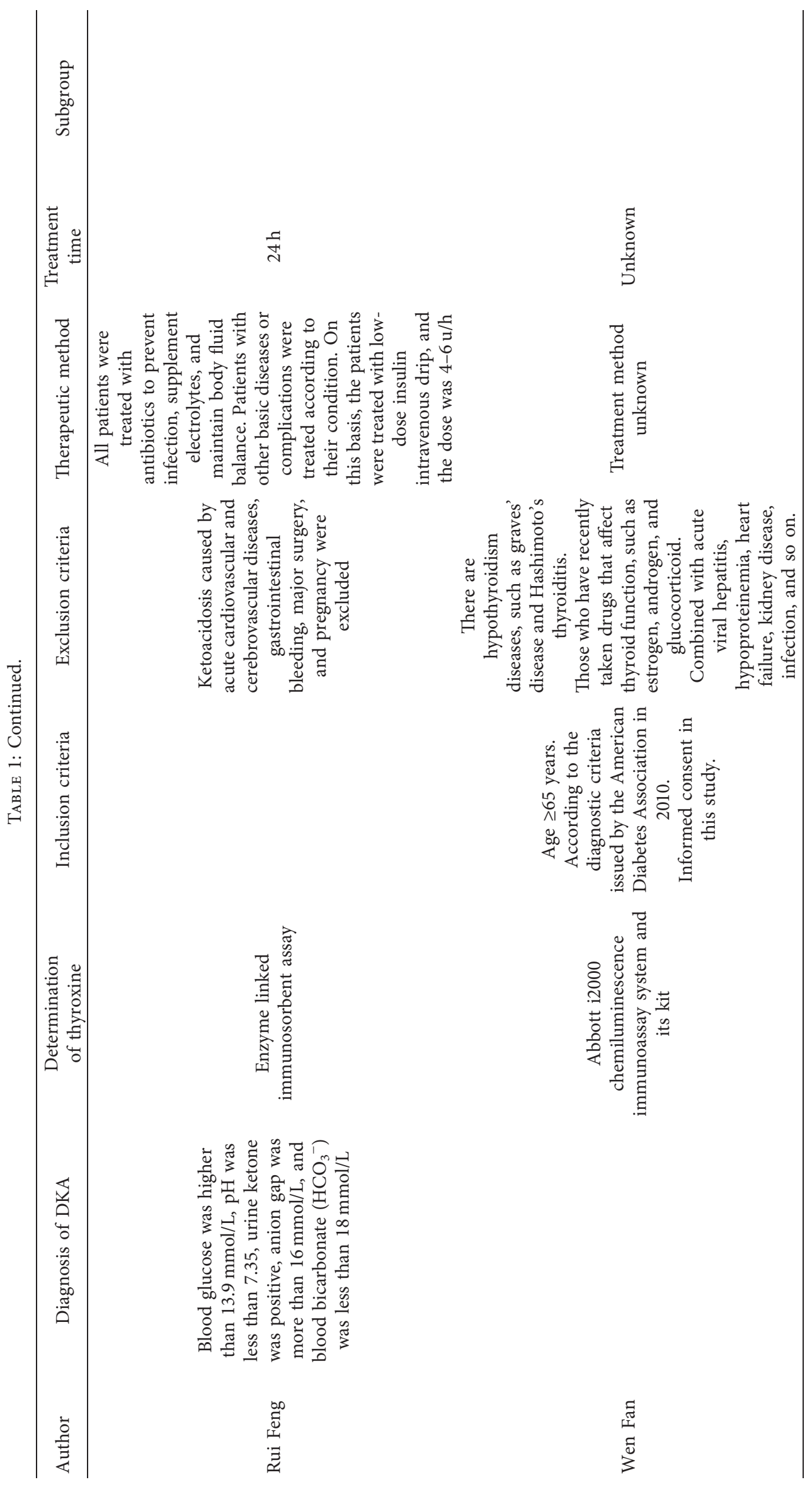




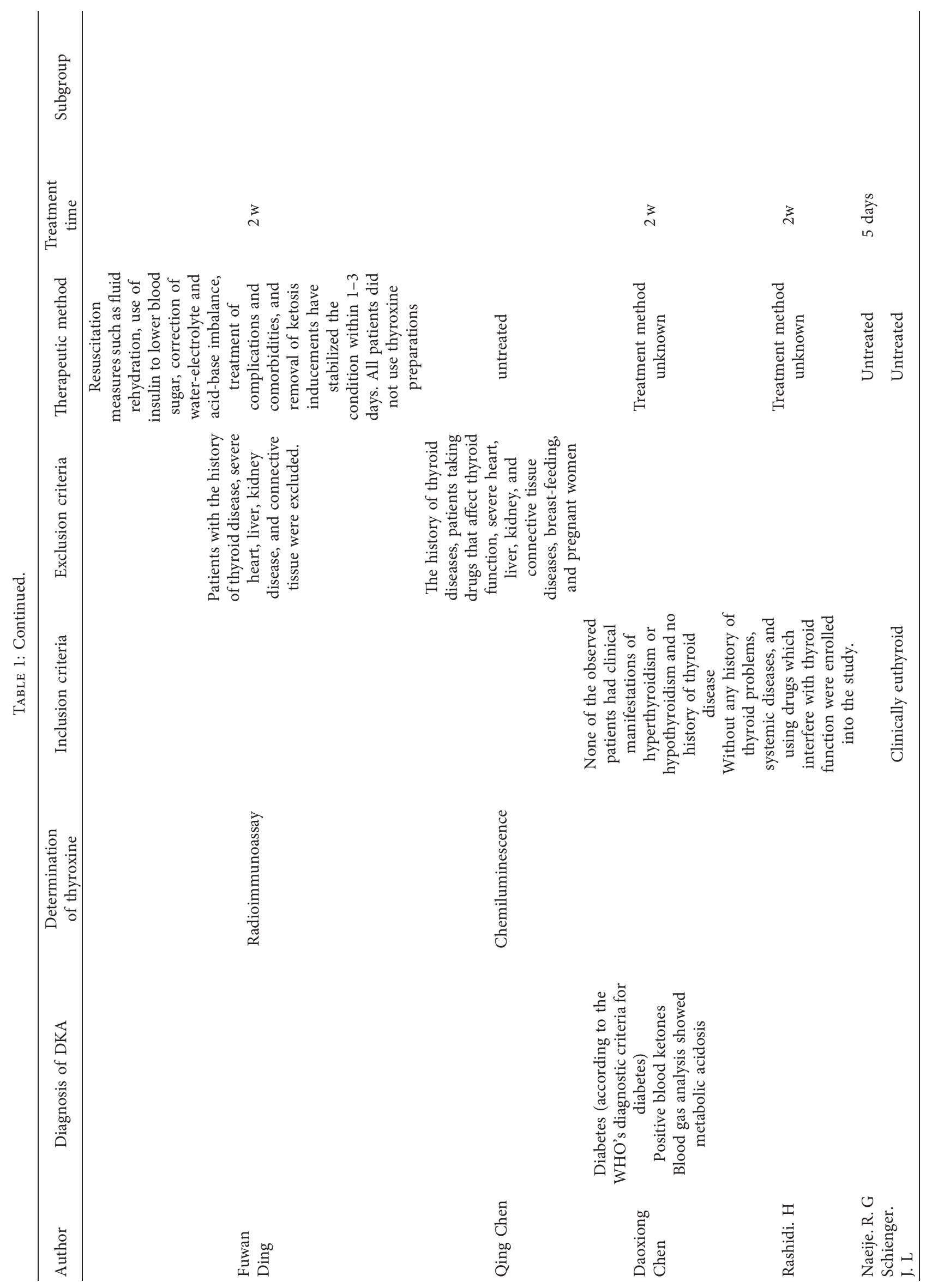




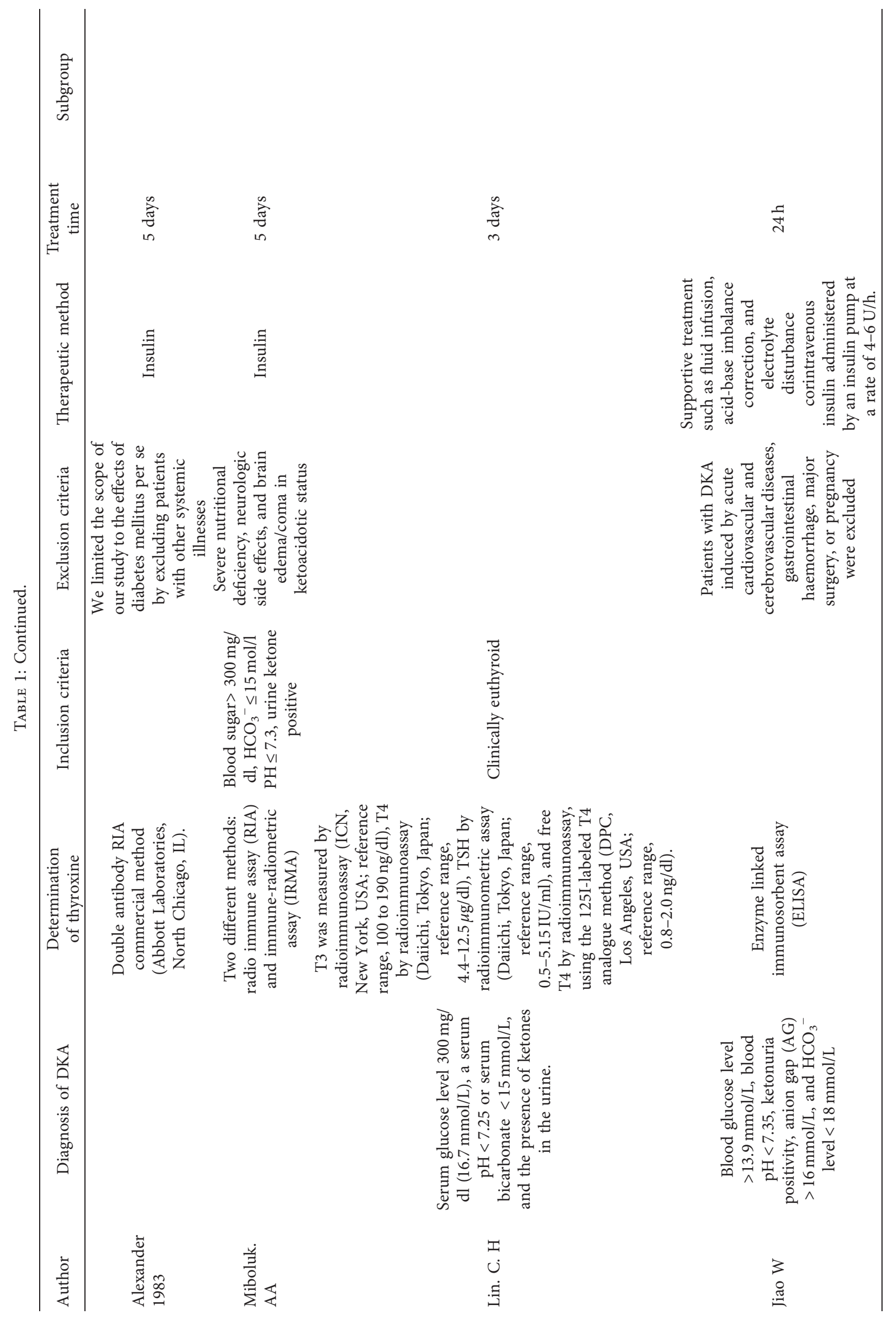




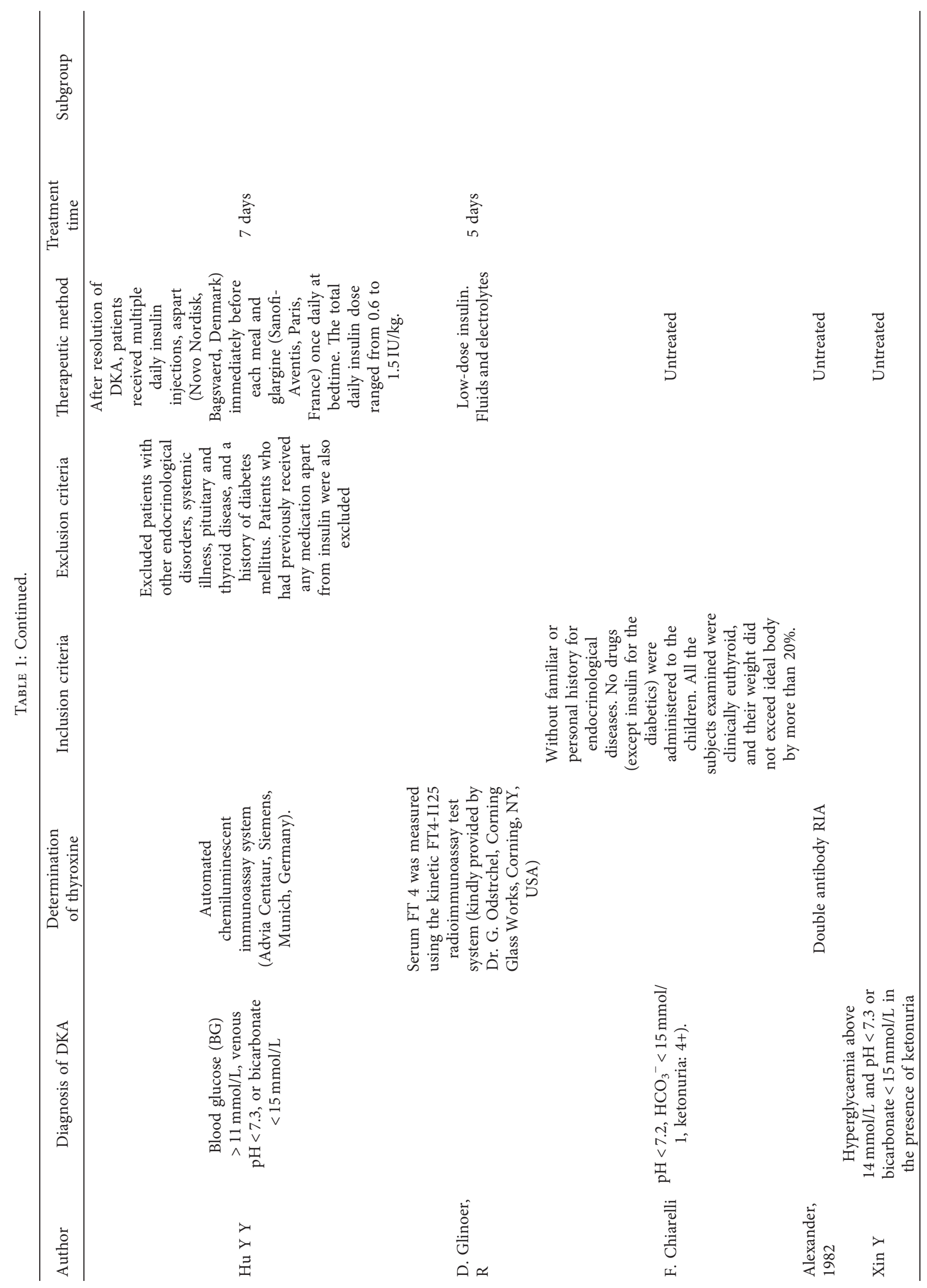




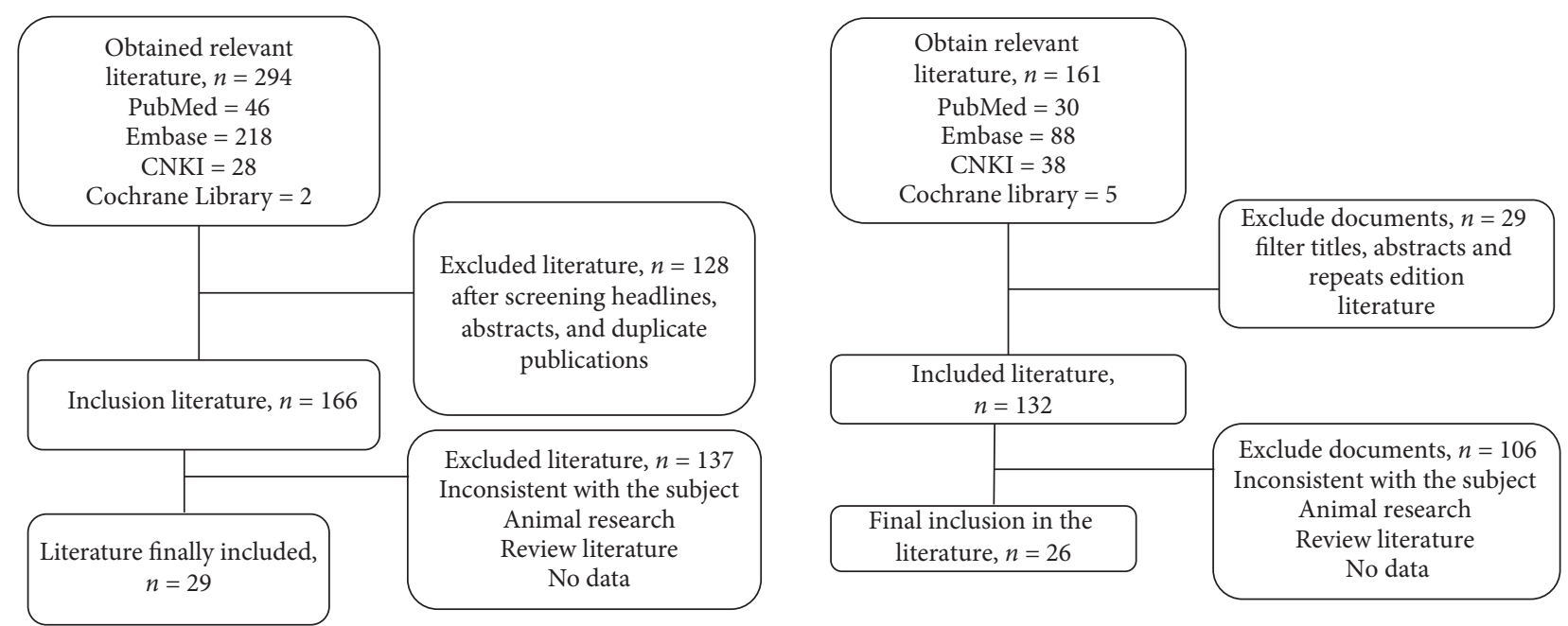

Figure 1: The process of study selection.

TABLE 2: Comparison of thyroid function before and after treatment in patients with diabetes and DKA.

\begin{tabular}{|c|c|c|c|c|c|c|c|c|}
\hline \multirow{2}{*}{ Author } & \multirow{2}{*}{ Year } & \multirow{2}{*}{ Country } & \multicolumn{3}{|c|}{ DKA } & \multicolumn{3}{|c|}{ After treatment } \\
\hline & & & Mean & SD & $n$ & Mean & SD & $n$ \\
\hline \multicolumn{9}{|l|}{$\mathrm{T} 4$} \\
\hline Daoxiong et al. [10] & 1999 & China & 79.38 & 19.13 & 65 & 78.32 & 16.5 & 65 \\
\hline Glinoer et al. [11] & 1980 & Belgium & 6.2 & 0.6 & 17 & 9.7 & 0.7 & 17 \\
\hline Wang [12] & 1999 & China & 110.26 & 45.89 & 62 & 118 & 48.57 & 62 \\
\hline Piao and Li [13] & 1999 & China & 71.4 & 12.6 & 8 & 70.9 & 12.3 & 8 \\
\hline Lin et al. [14] & 2003 & China & 4.39 & 3.03 & 76 & 7.72 & 2.29 & 76 \\
\hline Mirboluk et al. [15] & 2017 & Iran & 3.18 & 1.4 & 16 & 5.17 & 2.4 & 16 \\
\hline Naeije et al. [16] & 1978 & Germany & 5.7 & 3.05 & 19 & 8.9 & 2.18 & 19 \\
\hline Rashidi et al. [17] & 2017 & Iran & 7.6 & 2.53 & 20 & 8.41 & 2.51 & 20 \\
\hline Huang and Su [18] & 2016 & China & 96.41 & 5.12 & 63 & 110.34 & 8.32 & 63 \\
\hline Fan [19] & 2014 & China & 95.83 & 7.54 & 81 & 102.54 & 8.04 & 81 \\
\hline Wang and $\mathrm{Du}[20]$ & 2013 & China & 96.3 & 23.1 & 69 & 109 & 37.9 & 69 \\
\hline Zhang [21] & 2014 & China & 95.8 & 23.2 & 74 & 110.3 & 38.6 & 74 \\
\hline Ding and Ji [22] & 2011 & China & 96.29 & 20.1 & 30 & 102.3 & 20.55 & 30 \\
\hline Wang et al. [23] & 2018 & China & 65.68 & 20.32 & 40 & 90.33 & 20.95 & 40 \\
\hline \multicolumn{9}{|l|}{$\mathrm{T} 3$} \\
\hline Daoxiong et al. [10] & 1999 & China & 1 & 0.24 & 65 & 1.16 & 0.21 & 65 \\
\hline Wang [12] & 1999 & China & 1.26 & 0.46 & 62 & 2.29 & 0.59 & 62 \\
\hline Piao and Li [13] & 1999 & China & 51.43 & 3.51 & 8 & 82.37 & 2.58 & 8 \\
\hline Lin et al. [14] & 2003 & China & 59.36 & 36.11 & 76 & 140.63 & 48.24 & 76 \\
\hline Mirboluk et al. [15] & 2017 & Iran & 63.2 & 28.2 & 16 & 78.5 & 26.2 & 16 \\
\hline Naeije et al. [16] & 1978 & Germany & 37 & 6 & 19 & 105 & 9 & 19 \\
\hline Rashidi et al. [17] & 2017 & Iran & 86 & 25.7 & 20 & 161.25 & 38 & 20 \\
\hline Huang and Su [18] & 2016 & China & 1.34 & 0.25 & 63 & 1.65 & 0.31 & 63 \\
\hline Fan [19] & 2014 & China & 1.38 & 0.12 & 81 & 1.45 & 0.21 & 81 \\
\hline Wang and $\mathrm{Du}[20]$ & 2013 & China & 1.38 & 0.23 & 69 & 1.52 & 0.39 & 69 \\
\hline Zhang [21] & 2014 & China & 1.39 & 0.24 & 74 & 1.53 & 0.41 & 74 \\
\hline Ding and Ji [22] & 2011 & China & 0.75 & 0.2 & 30 & 1.68 & 0.33 & 30 \\
\hline Wang et al. [23] & 2018 & China & 0.84 & 0.3 & 40 & 1.55 & 0.33 & 40 \\
\hline \multicolumn{9}{|l|}{ FT4 } \\
\hline Daoxiong et al. [10] & 1999 & China & 14.21 & 2.8 & 65 & 13.8 & 2.5 & 65 \\
\hline Glinoer et al. [11] & 1980 & Belgium & 1.4 & 0.1 & 17 & 1.7 & 0.1 & 17 \\
\hline Hu et al. [24] & 2015 & China & 11.38 & 3.58 & 40 & 15.57 & 2.92 & 40 \\
\hline Jiao et al. [25] & 2016 & China & 11.61 & 3.53 & 120 & 14.23 & 3.01 & 120 \\
\hline Lin et al. [14] & 2003 & China & 0.59 & 0.36 & 76 & 1.29 & 0.32 & 76 \\
\hline Rashidi et al. [17] & 2017 & Iran & 1.07 & 0.43 & 20 & 1.58 & 0.62 & 20 \\
\hline
\end{tabular}


TABLE 2: Continued.

\begin{tabular}{|c|c|c|c|c|c|c|c|c|}
\hline \multirow{2}{*}{ Author } & \multirow{2}{*}{ Year } & \multirow{2}{*}{ Country } & \multicolumn{3}{|c|}{ DKA } & \multicolumn{3}{|c|}{ After treatment } \\
\hline & & & Mean & SD & $n$ & Mean & SD & $n$ \\
\hline Feng [26] & 2014 & China & 11.62 & 3.52 & 60 & 14.24 & 3.03 & 60 \\
\hline Huang and $\mathrm{Su}[18]$ & 2016 & China & 13.21 & 0.24 & 63 & 15.28 & 0.65 & 63 \\
\hline Fan $[19]$ & 2014 & China & 13.44 & 0.95 & 81 & 14.02 & 1.21 & 81 \\
\hline Wang and $\mathrm{Du}[20]$ & 2013 & China & 13 & 2.3 & 69 & 14.2 & 1 & 69 \\
\hline Zhang [21] & 2014 & China & 13.2 & 2.4 & 74 & 14.6 & 1.1 & 74 \\
\hline Ding and Ji [22] & 2011 & China & 13.32 & 2.52 & 30 & 13.92 & 3.99 & 30 \\
\hline Wang et al. [23] & 2018 & China & 11.91 & 2.85 & 40 & 14.26 & 2.47 & 40 \\
\hline \multicolumn{9}{|l|}{ FT3 } \\
\hline Daoxiong et al. [10] & 1999 & China & 2.48 & 0.9 & 65 & 3.38 & 0.98 & 65 \\
\hline Hu et al. [24] & 2015 & China & 2.63 & 0.58 & 40 & 4.77 & 1.15 & 40 \\
\hline Jiao et al. [25] & 2016 & China & 2.85 & 1.22 & 120 & 3.98 & 1.02 & 120 \\
\hline Rashidi et al. [17] & 2017 & Iran & 147 & 0.4 & 20 & 3.8 & 0.86 & 20 \\
\hline Feng [26] & 2014 & China & 2.87 & 1.23 & 60 & 3.96 & 1.03 & 60 \\
\hline Fan $[19]$ & 2014 & China & 3.54 & 0.23 & 81 & 3.6 & 0.34 & 81 \\
\hline Wang and $\mathrm{Du}[20]$ & 2013 & China & 3.54 & 0.53 & 69 & 3.69 & 0.51 & 69 \\
\hline Zhang [21] & 2014 & China & 3.55 & 0.54 & 74 & 3.65 & 0.48 & 74 \\
\hline Ding and Ji [22] & 2011 & China & 2.21 & 0.41 & 30 & 4.08 & 0.55 & 30 \\
\hline Wang et al. [23] & 2018 & China & 2.71 & 0.83 & 40 & 4.48 & 0.67 & 40 \\
\hline \multicolumn{9}{|l|}{ TSH } \\
\hline Daoxiong et al. [10] & 1999 & China & 1.95 & 0.85 & 65 & 1.74 & 0.87 & 65 \\
\hline Glinoer et al. [11] & 1980 & Belgium & 29 & 61 & 17 & 74 & 82 & 17 \\
\hline Hu et al. [24] & 2015 & China & 1.77 & 1.19 & 40 & 2.17 & 0.91 & 40 \\
\hline Jiao et al. [25] & 2016 & China & 1.8 & 0.76 & 120 & 2.33 & 0.87 & 120 \\
\hline Wang [12] & 1999 & China & 2.94 & 2.07 & 62 & 3.21 & 2.35 & 62 \\
\hline Lin et al. [14] & 2003 & China & 1.37 & 1.46 & 76 & 2.03 & 1.29 & 76 \\
\hline Mirboluk et al. [15] & 2017 & Iran & 1.85 & 1.5 & 16 & 1.79 & 1.3 & 16 \\
\hline Naeije et al. [16] & 1978 & Germany & 1.9 & 1.3 & 19 & 2.6 & 1.3 & 19 \\
\hline Feng [26] & 2014 & China & 1.82 & 0.75 & 60 & 2.32 & 0.86 & 60 \\
\hline Huang and Su [18] & 2016 & China & 4.48 & 1.24 & 63 & 4.09 & 1.03 & 63 \\
\hline Fan $[19]$ & 2014 & China & 4.08 & 0.28 & 81 & 3.89 & 0.43 & 81 \\
\hline Wang and $\mathrm{Du}[20]$ & 2013 & China & 4.47 & 1.59 & 69 & 4.12 & 1.47 & 69 \\
\hline Zhang [21] & 2014 & China & 4.49 & 1.61 & 74 & 4.24 & 1.51 & 74 \\
\hline Ding and Ji [22] & 2011 & China & 1.33 & 0.76 & 30 & 1.56 & 0.77 & 30 \\
\hline Wang et al. [23] & 2018 & China & 1.75 & 1.28 & 40 & 2.63 & 1.18 & 40 \\
\hline \multicolumn{9}{|l|}{ T3RU } \\
\hline Glinoer et al. [11] & 1980 & Belgium & 31.1 & 4.5 & 17 & 29.4 & 5.8 & 17 \\
\hline Lin et al. [14] & 2003 & China & 33.33 & 4.52 & 76 & 32.07 & 4.31 & 76 \\
\hline Mirboluk et al. [15] & 2017 & Iran & 32.4 & 1.8 & 16 & 32.1 & 1.5 & 16 \\
\hline Naeije et al. [16] & 1978 & Germany & 31.5 & 4.4 & 19 & 30.8 & 6.5 & 19 \\
\hline Rashidi et al. [17] & 2017 & Iran & 1.7 & 0.46 & 20 & 4.05 & 0.77 & 20 \\
\hline \multicolumn{9}{|l|}{ rT3 } \\
\hline Ding and Ji [22] & 2011 & China & 1.3 & 0.3 & 30 & 0.81 & 0.16 & 30 \\
\hline Naeije et al. [16] & 1978 & Germany & 40 & 26.15 & 19 & 24 & 26.15 & 19 \\
\hline Daoxiong et al. [10] & 1999 & China & 0.78 & 0.09 & 65 & 0.49 & 0.09 & 65 \\
\hline
\end{tabular}

estimator. It is more certain that the effect estimates obtained in the meta-analysis are effective. The funnel plot is shown in Figure 4.

3.2.2. Comparison of Thyroid Function before and after Treatment in Patients with Diabetes and DKA. 14 studies involved the comparison of T4 before and after treatment in patients with DKA, including a total of 640 patients with DkA; 13 studies involved the comparison of T3 before and after treatment, including a total of 623 patients with DkA; 13 studies involved the comparison of
FT4 before and after treatment, including a total of 755 patients with DkA; 10 studies involved the comparison of FT3 before and after treatment, including a total of 599 patients with DkA; 15 studies involved the comparison of TSH before and after treatment, including a total of 832 patients with DkA; 5 studies involved the comparison of T3RU before and after treatment, including a total of 148 patients with DkA; and 3 studies involved the comparison of rT3 before and after treatment, including a total of 114 patients with DkA. The results showed that patients with DKA had lower levels of T4, T3, FT4, and FT3 and higher level of rT3 compared with after treatment. The difference 
TABLE 3: Comparison of thyroid function between patients with diabetes with and without DKA.

\begin{tabular}{|c|c|c|c|c|c|c|c|c|}
\hline \multirow{2}{*}{ Author } & \multirow{2}{*}{ Year } & \multirow{2}{*}{ Country } & \multicolumn{3}{|c|}{ DKA } & \multicolumn{3}{|c|}{ Control } \\
\hline & & & Mean & $\mathrm{SD}$ & $n$ & Mean & $\mathrm{SD}$ & $n$ \\
\hline \multicolumn{9}{|l|}{$\mathrm{T} 4$} \\
\hline Alexander et al. [27] & 1983 & United States & 5.5 & 0.6 & 12 & 8.7 & 0.6 & 6 \\
\hline Chiarelli et al.[28] & 1989 & Germany & 58.22 & 15.02 & 16 & 74.04 & 23.07 & 45 \\
\hline Daoxiong et al. [10] & 1999 & China & 79.38 & 19.13 & 65 & 94.6 & 18.12 & 60 \\
\hline Li et al. [29] & 2012 & China & 82.4289 & 22.6743 & 38 & 109.094 & 17.9297 & 36 \\
\hline Lin et al. [14] & 2003 & China & 4.39 & 3.03 & 76 & 7.6 & 1.86 & 62 \\
\hline Schienger et al. [30] & 1982 & Germany & 7.6 & 0.3 & 8 & 7.8 & 0.5 & 8 \\
\hline Huang and $\mathrm{Su}[18]$ & 2016 & China & 96.41 & 5.12 & 63 & 107.52 & 8.12 & 62 \\
\hline Fan $[19]$ & 2014 & China & 95.83 & 7.54 & 81 & 106.45 & 9.09 & 94 \\
\hline Zhao et al. [31] & 2012 & China & 5.65 & 2.8 & 91 & 9.28 & 2.85 & 110 \\
\hline Wang and $\mathrm{Du}[20]$ & 2013 & China & 96.3 & 23.1 & 69 & 114.8 & 41.2 & 74 \\
\hline Qiao [32] & 2012 & China & 5.95 & 1.57 & 40 & 7.8 & 1.67 & 40 \\
\hline S. Liu [33] & 2016 & China & 92.9 & 18.78 & 23 & 114.09 & 18.83 & 31 \\
\hline Chen et al. [34] & 2016 & China & 102.46 & 22.73 & 65 & 107.28 & 23.28 & 65 \\
\hline Zhang [21] & 2014 & China & 95.8 & 23.2 & 74 & 165 & 42.3 & 84 \\
\hline Ding and Ji [22] & 2011 & China & 96.29 & 20.1 & 30 & 100.3 & 20.33 & 30 \\
\hline \multicolumn{9}{|l|}{ T3 } \\
\hline Alexander et al. [27] & 1983 & United States & 49.9 & 7.3 & 12 & 88 & 8 & 6 \\
\hline Alexander et al. [6] & 1982 & United States & 49 & 9 & 4 & 103 & 7 & 11 \\
\hline Chiarelli et al.[28] & 1989 & Germany & 1.04 & 0.36 & 16 & 1.39 & 0.42 & 45 \\
\hline Daoxiong et al. [10] & 1999 & China & 1 & 0.24 & 65 & 1.25 & 0.36 & 60 \\
\hline Li et al. [29] & 2012 & China & 1.5482 & 0.4371 & 38 & 1.9497 & 0.2762 & 36 \\
\hline Lin et al. [14] & 2003 & & 59.36 & 36.11 & 76 & 91.4 & 31.85 & 62 \\
\hline Schienger et al. [30] & 1982 & Germany & 124 & 14 & 8 & 115 & 6 & 8 \\
\hline Huang and $\mathrm{Su}[18]$ & 2016 & China & 1.34 & 0.25 & 63 & 1.52 & 0.31 & 62 \\
\hline Fan $[19]$ & 2014 & China & 1.38 & 0.12 & 81 & 1.55 & 0.21 & 94 \\
\hline Zhao et al. [31] & 2012 & China & 0.54 & 0.51 & 91 & 1.02 & 0.38 & 110 \\
\hline Wang and Du [20] & 2013 & China & 1.38 & 0.23 & 69 & 1.59 & 0.47 & 74 \\
\hline Qiao [32] & 2012 & China & 0.75 & 0.22 & 40 & 1.05 & 0.21 & 40 \\
\hline S. Liu [33] & 2016 & China & 1.6 & 0.41 & 23 & 1.85 & 0.33 & 31 \\
\hline Chen et al. [34] & 2016 & China & 1.53 & 0.24 & 65 & 1.72 & 0.27 & 65 \\
\hline Zhang [21] & 2014 & China & 1.39 & 0.24 & 74 & 1.61 & 0.45 & 84 \\
\hline Ding and Ji [22] & 2011 & & 0.75 & 0.2 & 30 & 1.72 & 0.31 & 30 \\
\hline \multicolumn{9}{|l|}{ FT4 } \\
\hline Liu [35] & 2012 & China & 0.84 & 0.21 & 20 & 1.15 & 0.38 & 60 \\
\hline Chiarelli et al.[28] & 1989 & Germany & 10.24 & 2.94 & 16 & 11.55 & 3.62 & 45 \\
\hline Daoxiong et al. [10] & 1999 & China & 14.21 & 2.8 & 65 & 12.13 & 2.88 & 60 \\
\hline Qiu et al. [36] & 2018 & China & 12.4 & 4.89 & 75 & 15.97 & 3.08 & 39 \\
\hline Lin et al. [14] & 2003 & China & 0.59 & 0.36 & 76 & 1.18 & 0.4 & 62 \\
\hline Huang and $\mathrm{Su}[18]$ & 2016 & China & 13.21 & 0.24 & 63 & 14.29 & 0.31 & 62 \\
\hline Fan $[19]$ & 2014 & China & 13.44 & 0.95 & 81 & 14.35 & 0.36 & 94 \\
\hline Xin et al. [37] & 2010 & China & 12.99 & 7.3 & 85 & 15.34 & 3.97 & 118 \\
\hline Wang and Du [20] & 2013 & China & 13 & 2.3 & 69 & 14 & 1.2 & 74 \\
\hline Qiao [32] & 2012 & China & 1.13 & 0.26 & 40 & 1.21 & 0.17 & 40 \\
\hline S. Liu [33] & 2016 & China & 10.76 & 2.1 & 23 & 12.12 & 1.46 & 31 \\
\hline Chen et al. [34] & 2016 & China & 11.86 & 2.57 & 65 & 12.51 & 2.78 & 65 \\
\hline Zhang [21] & 2014 & China & 13.2 & 2.4 & 74 & 14.9 & 1.3 & 84 \\
\hline Ding and Ji [22] & 2011 & China & 13.32 & 2.52 & 30 & 14.31 & 4.01 & 30 \\
\hline Schienger et al. [30] & 1982 & Germany & 2.3 & 0.29 & 8 & 2.2 & 0.29 & 8 \\
\hline \multicolumn{9}{|l|}{ FT3 } \\
\hline Liu [35] & 2012 & China & 3.96 & 0.92 & 20 & 5.83 & 1.96 & 60 \\
\hline Chiarelli et al.[28] & 1989 & Germany & 2.05 & 1.01 & 16 & 2.35 & 0.71 & 45 \\
\hline Daoxiong et al. [10] & 1999 & China & 2.48 & 0.9 & 65 & 3.46 & 0.89 & 60 \\
\hline Qiu et al. [36] & 2018 & China & 2.47 & 0.74 & 75 & 3.07 & 0.91 & 39 \\
\hline Fan [19] & 2014 & China & 3.54 & 0.23 & 81 & 3.69 & 0.44 & 94 \\
\hline Xin et al. [37] & 2010 & China & 2.61 & 1.93 & 85 & 3.31 & 1.27 & 118 \\
\hline Wang and Du [20] & 2013 & China & 3.54 & 0.53 & 69 & 3.65 & 0.49 & 74 \\
\hline Qiao [32] & 2012 & China & 2.21 & 0.61 & 40 & 2.85 & 0.3 & 40 \\
\hline
\end{tabular}


TABLE 3: Continued.

\begin{tabular}{|c|c|c|c|c|c|c|c|c|}
\hline \multirow{2}{*}{ Author } & \multirow{2}{*}{ Year } & \multirow{2}{*}{ Country } & \multicolumn{3}{|c|}{ DKA } & \multicolumn{3}{|c|}{ Control } \\
\hline & & & Mean & $\mathrm{SD}$ & $n$ & Mean & SD & $n$ \\
\hline S. Liu [33] & 2016 & China & 4.32 & 0.66 & 23 & 4.95 & 0.63 & 31 \\
\hline Chen et al. [34] & 2016 & China & 3.95 & 1.14 & 65 & 4.46 & 1.17 & 65 \\
\hline Zhang [21] & 2014 & China & 3.55 & 0.54 & 74 & 3.64 & 0.51 & 84 \\
\hline Ding and Ji [22] & 2011 & China & 2.21 & 0.41 & 30 & 4.25 & 0.41 & 30 \\
\hline \multicolumn{9}{|l|}{ TSH } \\
\hline Alexander et al. [27] & 1983 & The United States & 3.4 & 0.9 & 12 & 4.4 & 0.7 & 6 \\
\hline Liu [35] & 2012 & China & 0.83 & 0.73 & 20 & 1.25 & 1.19 & 60 \\
\hline Chiarelli et al.[28] & 1989 & Germany & 1.66 & 0.69 & 16 & 2.56 & 1.27 & 45 \\
\hline Daoxiong et al. [10] & 1999 & China & 1.95 & 0.85 & 65 & 2.02 & 0.96 & 60 \\
\hline Li et al. [29] & 2012 & China & 1.5092 & 1.3515 & 38 & 2.0213 & 0.9604 & 36 \\
\hline Lin et al. [14] & 2003 & China & 1.37 & 1.46 & 76 & 1.6 & 1.01 & 62 \\
\hline Huang and $\mathrm{Su}[18]$ & 2016 & China & 4.48 & 1.24 & 63 & 4.46 & 1.31 & 62 \\
\hline Fan $[19]$ & 2014 & China & 4.08 & 0.28 & 81 & 3.95 & 0.23 & 94 \\
\hline Xin et al. [37] & 2010 & China & 1.7 & 1.48 & 85 & 1.66 & 0.77 & 118 \\
\hline Zhao et al. [31] & 2012 & China & 2.49 & 2.73 & 91 & 2.45 & 2.01 & 110 \\
\hline Wang and Du [20] & 2013 & China & 4.47 & 1.59 & 69 & 4.23 & 1.53 & 74 \\
\hline Qiao [32] & 2012 & China & 1.38 & 0.86 & 40 & 1.82 & 0.88 & 40 \\
\hline S. Liu [33] & 2016 & China & 1.27 & 1.04 & 23 & 2.17 & 1.33 & 31 \\
\hline Chen et al. [34] & 2016 & China & 3.15 & 0.58 & 65 & 3.17 & 0.71 & 65 \\
\hline Zhang [21] & 2014 & China & 4.49 & 1.61 & 74 & 4.23 & 1.5 & 84 \\
\hline Ding and Ji [22] & 2011 & China & 1.33 & 0.76 & 30 & 1.43 & 0.82 & 30 \\
\hline \multicolumn{9}{|l|}{ rT3 } \\
\hline Ding and Ji [22] & 2011 & China & 1.3 & 0.3 & 30 & 0.83 & 0.17 & 30 \\
\hline Chiarelli et al.[28] & 1989 & Germany & 0.23 & 0.1 & 16 & 0.22 & 0.07 & 45 \\
\hline Alexander et al. [27] & 1983 & The United States & 57.8 & 25.3 & 12 & 35.7 & 8.3 & 6 \\
\hline Schienger.J.B & 1982 & Germany & 25.2 & 3.4 & 8 & 23.5 & 5.4 & 8 \\
\hline Alexander et al. [6] & 1982 & The United States & 83 & 2 & 4 & 33 & 2 & 35 \\
\hline Daoxiong et al. [10] & 1999 & China & 0.78 & 0.09 & 65 & 0.52 & 0.1 & 70 \\
\hline
\end{tabular}

was statistically significant (T4:I2 $=86.2 \%, p<0.01$, $Z=4.50, p<0.01, \mathrm{SMD}=-0.742,95 \% \mathrm{CI}:-1.066$ to 0.419 ; T3 : I2 =93\%, $p<0.01, Z=6.04, p<0.01, \mathrm{SMD}=-1.538$, $95 \% \mathrm{CI}:-2.037$ to $-1.039 ; \mathrm{FT} 4: \mathrm{I} 2=93.8 \%, p<0.01$, $Z=4.52, \quad p<0.01, \quad \mathrm{SMD}=-1.035,95 \% \mathrm{CI}:-1.483$ to $-0.586 ; \quad$ FT3 : I2 $=95.9 \%, \quad p<0.01, \quad Z=3.68, \quad p<0.01$, $\mathrm{SMD}=-1.258,95 \% \mathrm{CI}:-1.926$ to $-0.589 ;$ rT3 $: \mathrm{I} 2=94.7 \%$, $p<0.01, Z=2.57, p=0.01, \mathrm{SMD}=1.967,95 \% \mathrm{CI}: 0.467$ to 3.467 Figure 5). There was no significant difference in TSH and T3RU in patients with DKA before and after treatment. Egger's test (T4, $p=0.566$; T3RU, $p=0.243$; FT4, $p=0.175$; FT3, $p=0.988$; TSH, 0.599; rT3, $p=0.236$ ) showed that there was no obvious publication bias, further analysis by the trim-and-fill method showed that the publication bias (T3, $p=0.006$ ) did not affect the estimator, and it was more certain that the effect estimation obtained in the meta-analysis was effective. The funnel plot is shown in Figure 6.

3.2.3. Comparison of Severity of DKA and Thyroid Function in Patients with Diabetes and DKA. Three studies involved the comparison of the severity of DKA with thyroid function. The results showed that as the degree of DKA aggravated, the levels of T4, T3, FT4, and FT3 further decreased. The level of TSH increased with the aggravation of DKA, but it was not statistically significant (Figure 7).

\section{Discussion}

This meta-analysis study showed that the levels of T4, T3, FT3, FT4, and TSH were lower and the level of rT3 was higher in patients with DKA compared with patients with diabetes but not DKA. The levels of T4, T3, FT3, and FT4 were lower and the level of rT3 was higher compared with after treatment in patients with diabetes and DKA. As the aggravation of DKA, the levels of T4, T3, FT3, and FT4 would further decrease, but there was no statistical difference in the change of TSH.

DKA can affect the function of the hypothalamus-pituitary-thyroid axis directly or indirectly due to various factors such as relatively insufficient insulin secretion and metabolic disorders, thus affecting thyroid function [38]. Piconi et al. found that large blood glucose fluctuations trigger the production of nitrotyrosine and induce the expression of adhesion molecules and IL-6 [39]. The release of a large number of cytokines acted on the hypothalamuspituitary-thyroid axis through a variety of ways, which can also affect the synthesis, secretion, metabolism, and feedback of thyroid hormones [40]. An increase in cytokines such as IL-6 synchronizing with a low T3 level is often observed which may cause hypothalamus involvement [41]. The body's caloric intake is seriously insufficient in patients with DKA, leading to hypoxia in the cells, which reduced the biological activity of $5^{\prime}$-deiodinase, resulting in a significant 
$\mathrm{T} 4$

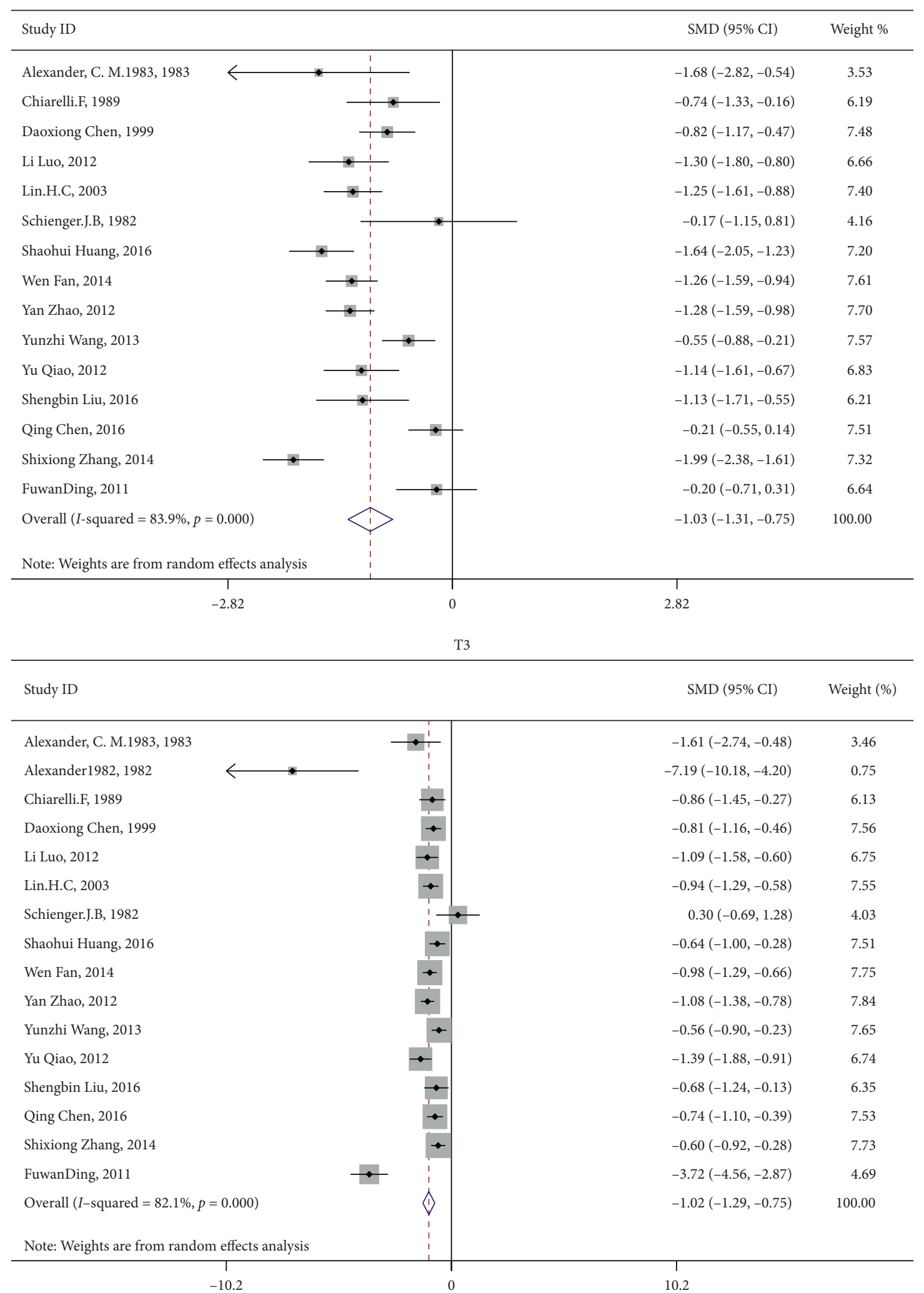

(a)

Figure 2: Continued. 
FT4

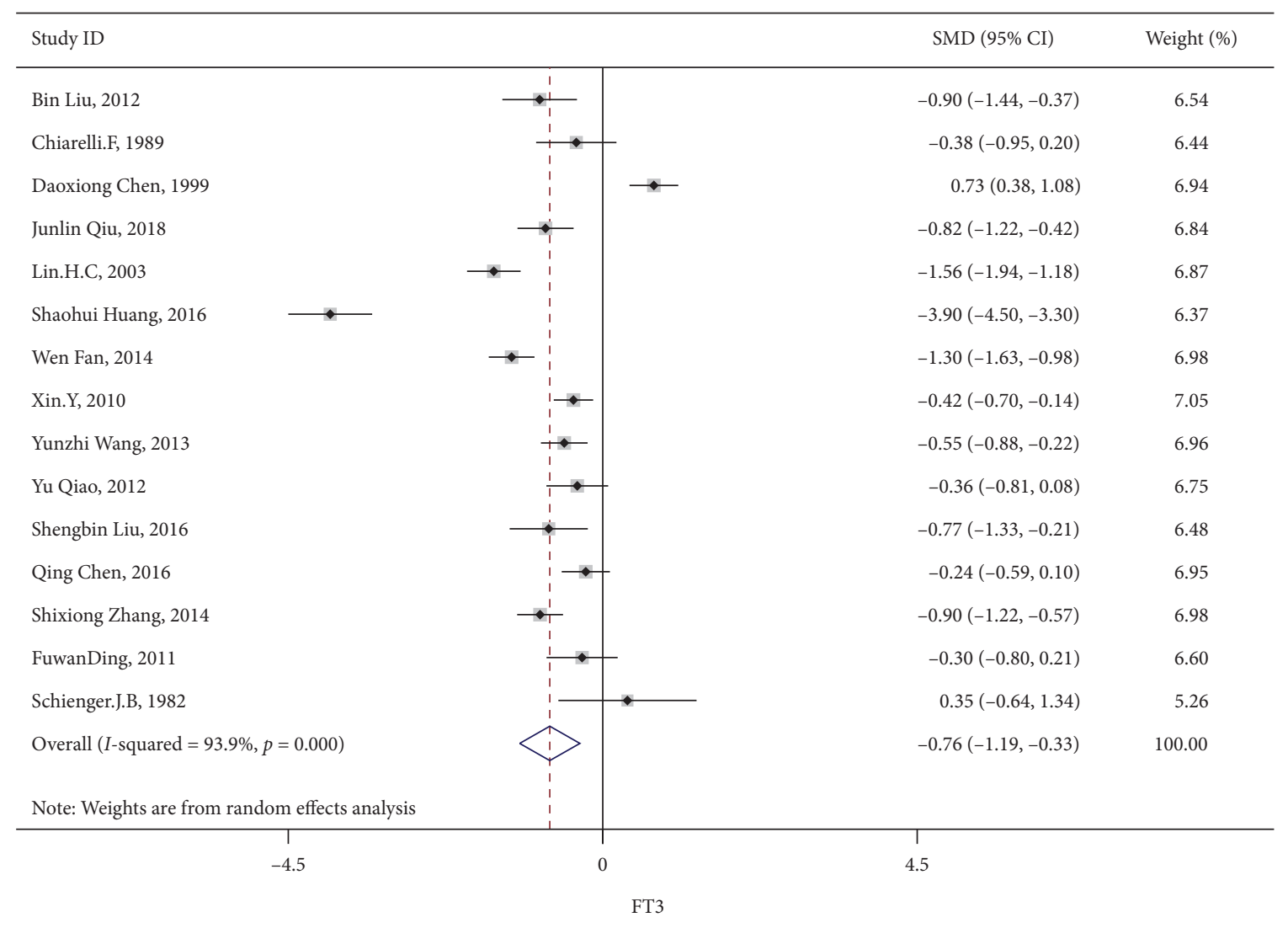

\begin{tabular}{|c|c|c|c|}
\hline Study ID & & SMD $(95 \% \mathrm{CI})$ & Weight (\%) \\
\hline Bin Liu, 2012 & $\because$ & $-1.07(-1.61,-0.53)$ & 7.97 \\
\hline Chiarelli.F, 1989 & $\rightarrow$ & $-0.38(-0.95,0.20)$ & 7.79 \\
\hline Daoxiong Chen, 1999 & $\rightarrow \frac{1}{1}$ & $-1.10(-1.46,-0.73)$ & 8.85 \\
\hline Junlin Qiu, 2018 & & $-0.75(-1.15,-0.35)$ & 8.69 \\
\hline Wen Fan, 2014 & & $-0.42(-0.72,-0.12)$ & 9.11 \\
\hline Xin.Y, 2010 & & $-0.44(-0.73,-0.16)$ & 9.18 \\
\hline Yunzhi Wang, 2013 & $\rightarrow$ & $-0.22(-0.54,0.11)$ & 9.00 \\
\hline Yu Qiao, 2012 & $\rightarrow$ & $-1.33(-1.82,-0.85)$ & 8.26 \\
\hline Shengbin Liu, 2016 & & $-0.98(-1.55,-0.41)$ & 7.81 \\
\hline Qing Chen, 2016 & $i \rightarrow$ & $-0.44(-0.79,-0.09)$ & 8.92 \\
\hline Shixiong Zhang, 2014 & $\rightarrow$ & $-0.17(-0.48,0.14)$ & 9.06 \\
\hline FuwanDing, 2011 & & $-4.98(-6.01,-3.94)$ & 5.37 \\
\hline Overall $(I$-squared $=89.6 \%, p=0.000)$ & & $-0.88(-1.24,-0.52)$ & 100.00 \\
\hline \multicolumn{4}{|l|}{ Note: Weights are from random effects analysis } \\
\hline-6.01 & c & 6.01 & \\
\hline
\end{tabular}

(b)

Figure 2: Continued. 
rT3

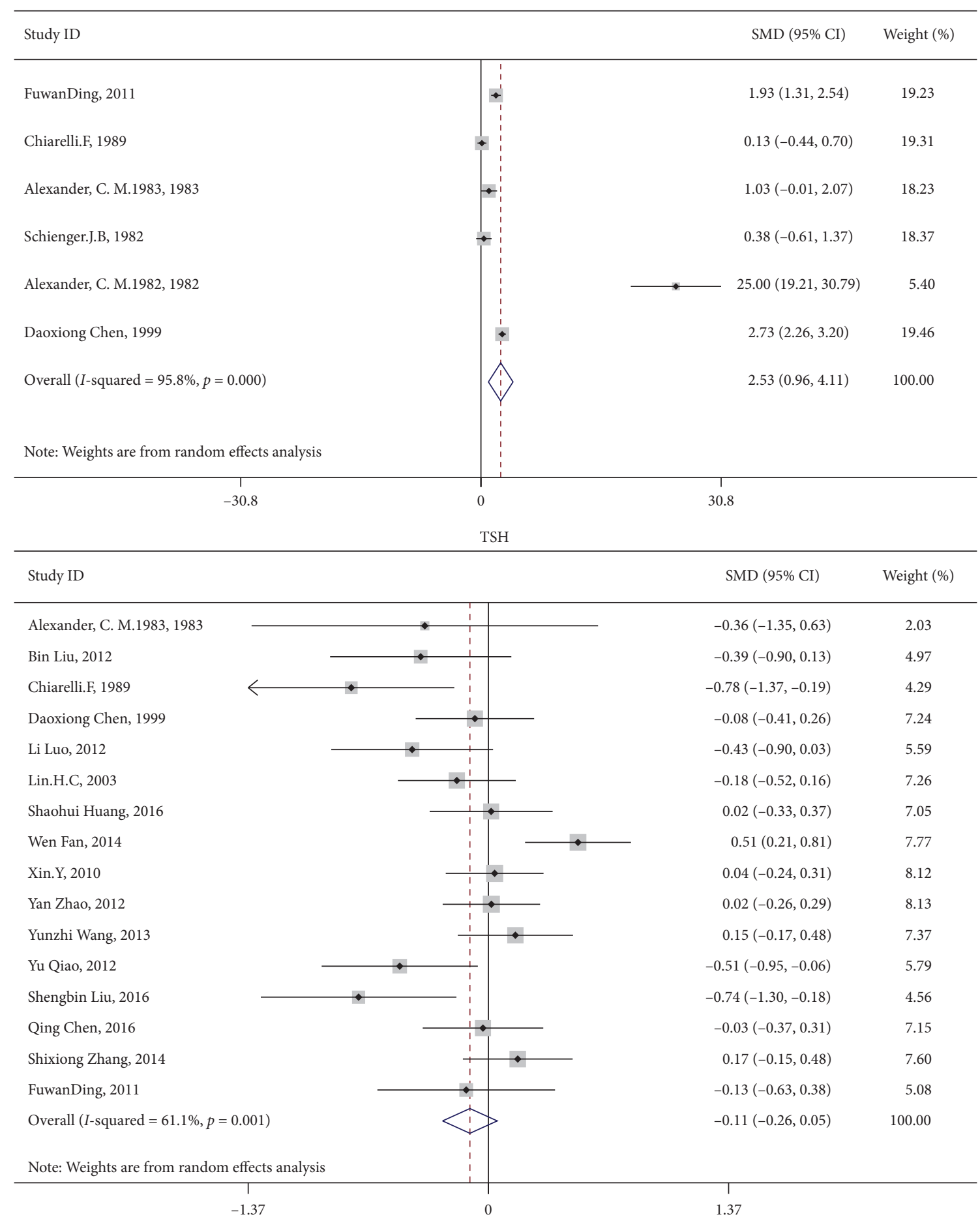

(c)

FIGURE 2: Forest plot of T4, T3, FT4, FT3, rT3, and TSH compared with patients with DKA and diabetes. 


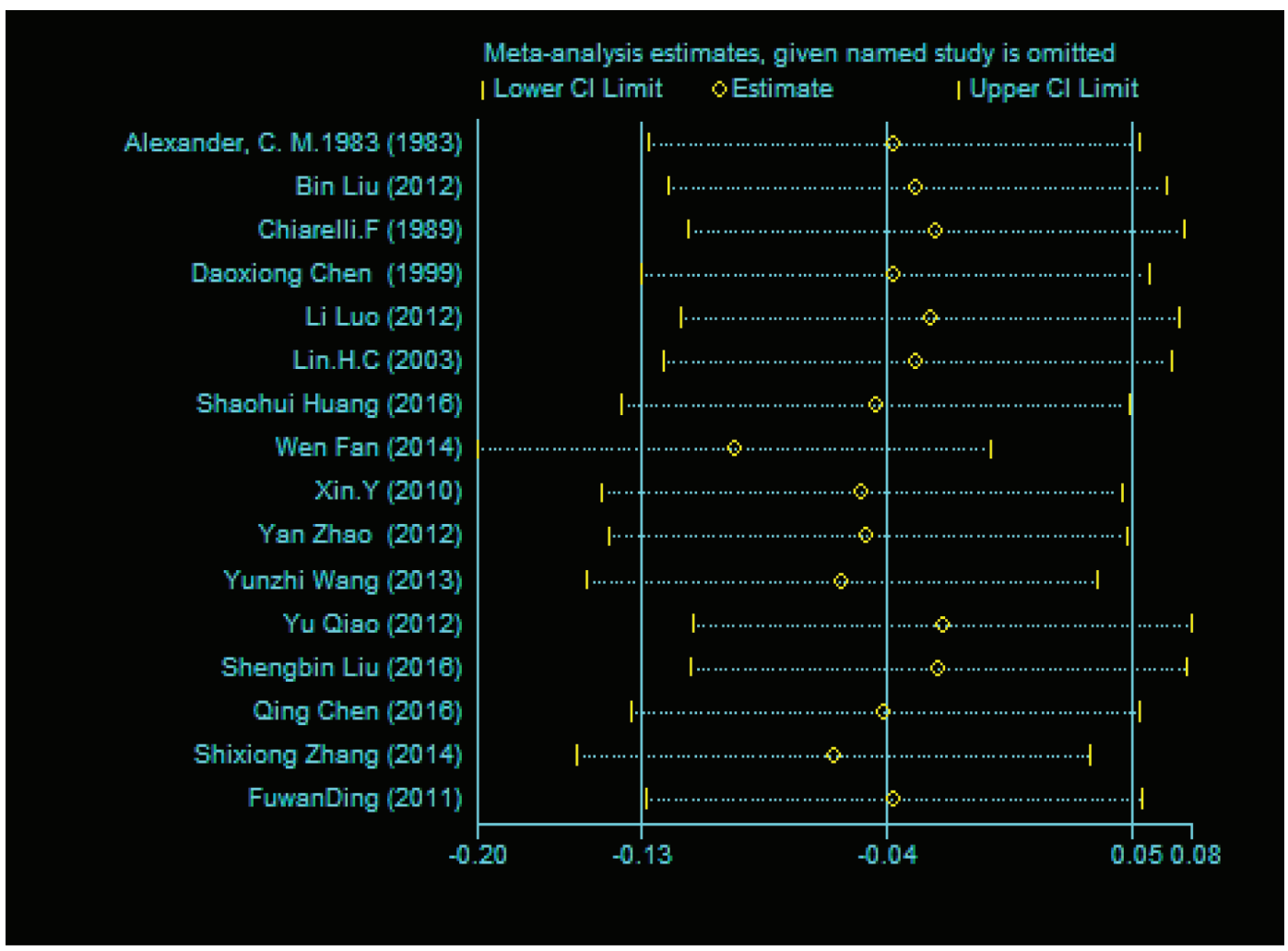

\begin{tabular}{l} 
Study ID \\
\hline Alexander, C. M.1983, 1983 \\
Bin Liu, 2012 \\
Chiarelli.F, 1989 \\
Daoxiong Chen, 1999 \\
Li Luo, 2012 \\
Lin.H.C, 2003 \\
Shaohui Huang, 2016 \\
Xin.Y, 2010 \\
Yan Zhao, 2012 \\
Yunzhi Wang, 2013 \\
Yu Qiao, 2012 \\
Shengbin Liu, 2016 \\
Qing Chen, 2016 \\
Shixiong Zhang, 2014 \\
FuwanDing, 2011 \\
Overall (I-squared = 42.6\%, $p=0.041)$ \\
Note: Weights are from random effects analysis
\end{tabular}

Figure 3: Sensitivity analysis of TSH. 


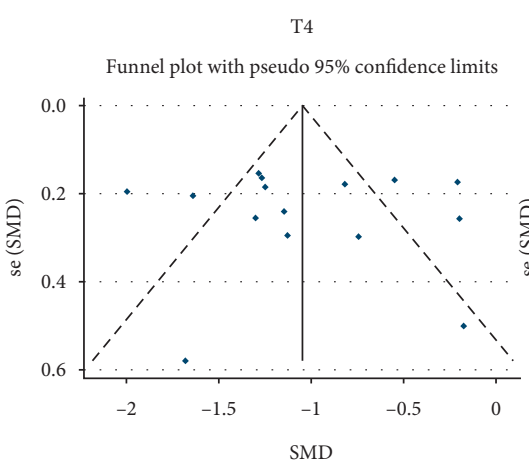

FT3

Funnel plot with pseudo 95\% confidence limits

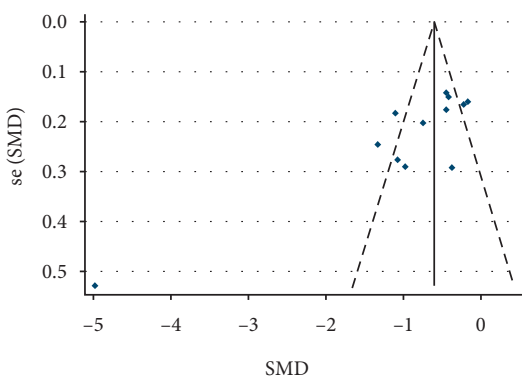

T3

Funnel plot with pseudo $95 \%$ confidence limits

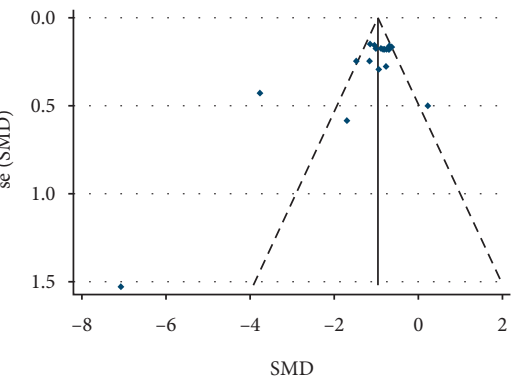

rT3

Funnel plot with pseudo 95\% confidence limits

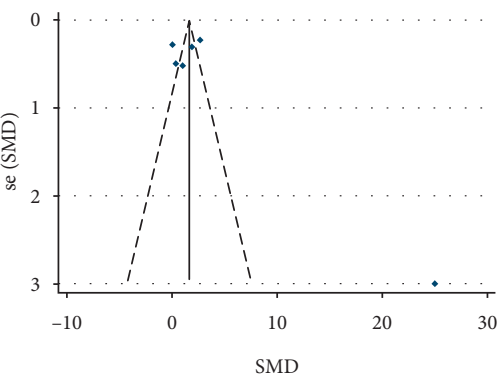

FT4

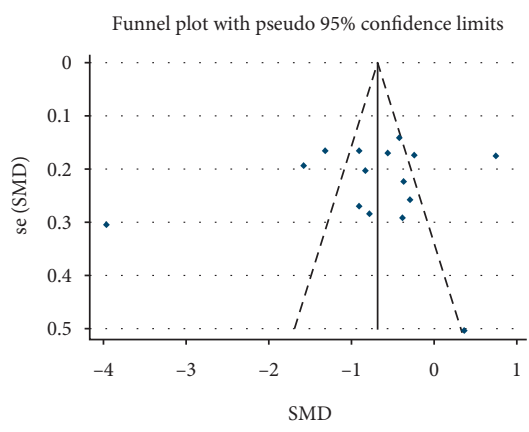

TSH

Funnel plot with pseudo 95\% confidence limits

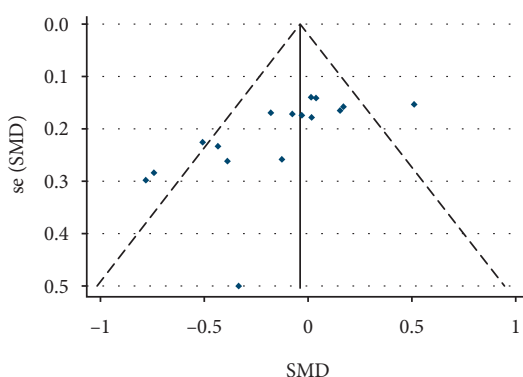

Figure 4: Funnel plot of T4, T3, FT4, FT3, rT3, and TSH compared with patients with DKA and diabetes.

reduction in the conversion of $\mathrm{T} 4$ to $\mathrm{T} 3$, and a significant reduction in the levels and activity of thyroid hormones [42]. Studies have shown that T1DM and thyroid diseases have a common genetic basis [43]. There is a significant positive correlation between serum TSH and antithyroid antibodies (TRAb, TPOAb, and TGAb) in patients with T2DM, suggesting that abnormal thyroid function in patients with T2DM is autoimmune-mediated pathogenesis [44].

Studies also found that the severity of impaired hypothalamus-hypophysial-thyroid regulation seems to be related to the degree of metabolic disorders regardless of the presence of antithyroid antibodies [45]. Previous studies have shown that the levels of serum $\mathrm{T} 3$ and $\mathrm{T} 4$ are related to the severity of the disease [46, 47]. Similarly, Balsamo et al. showed that changes in hormone levels are usually related to the severity of metabolic disorders, among which thyroid function is one of the most serious disorders. The hypothalamus-pituitary-thyroid axis showed variable damage, which was defined as nonthyroid disease syndrome (NTIS) [45]. The relationship between the degree of NTIS and the severity of metabolic disorders has previously been reported in adults and children [48-51]. NTIS is now more commonly used to describe a typical change in the serum levels of thyroid-related hormones that may occur after an acute or chronic disease not caused by intrinsic abnormalities in thyroid function. Changes in the hypothalamic-pituitary- thyroid axis also occur in diseases, usually associated with low levels of T3, which gave rise to the term "low T3 syndrome" [52].

It was now well known that most circulating T3 and almost all rT3 came from the peripheral deiodination of T4 $[53,54]$. Pittman et al. found that DKA played a certain role in the peripheral transformation of T4 [55]. The moderate decrease in serum T4 observed in patients with DKA has been described previously, which was corrected after treatment, and it seemed to be due to acquired deficiency of T4 binding to serum protein [56]. The factors of dietary, especially carbohydrates, played an important role in the regulation of $\mathrm{T} 3[57,58]$. The presence of carbohydrate deprivation in DKA seemed to rapidly inhibit the deiodination of $\mathrm{T} 4$ by type 1 iodothyronine-deiodinase in the liver, thereby inhibiting the production of $\mathrm{T} 3$ and preventing the metabolism of rT3 [59]. Carbohydrate deprivation will lead to a decrease in basal metabolic rate. The decrease in thyroid hormones is represented the body's remaining adaptive response to calories and protein by inducing hypothyroidism theoretically [60]. It was reported that the average level of rT3 was increased in patients with insulin-dependent diabetes, and the average metabolic clearance rate of rT3 is decreased $[55,61]$. The result of Pittman CS et al. suggested that T4 monodeiodination of both phenyl rings was significantly impaired in uncontrolled diabetes, and they 
$\mathrm{T} 4$

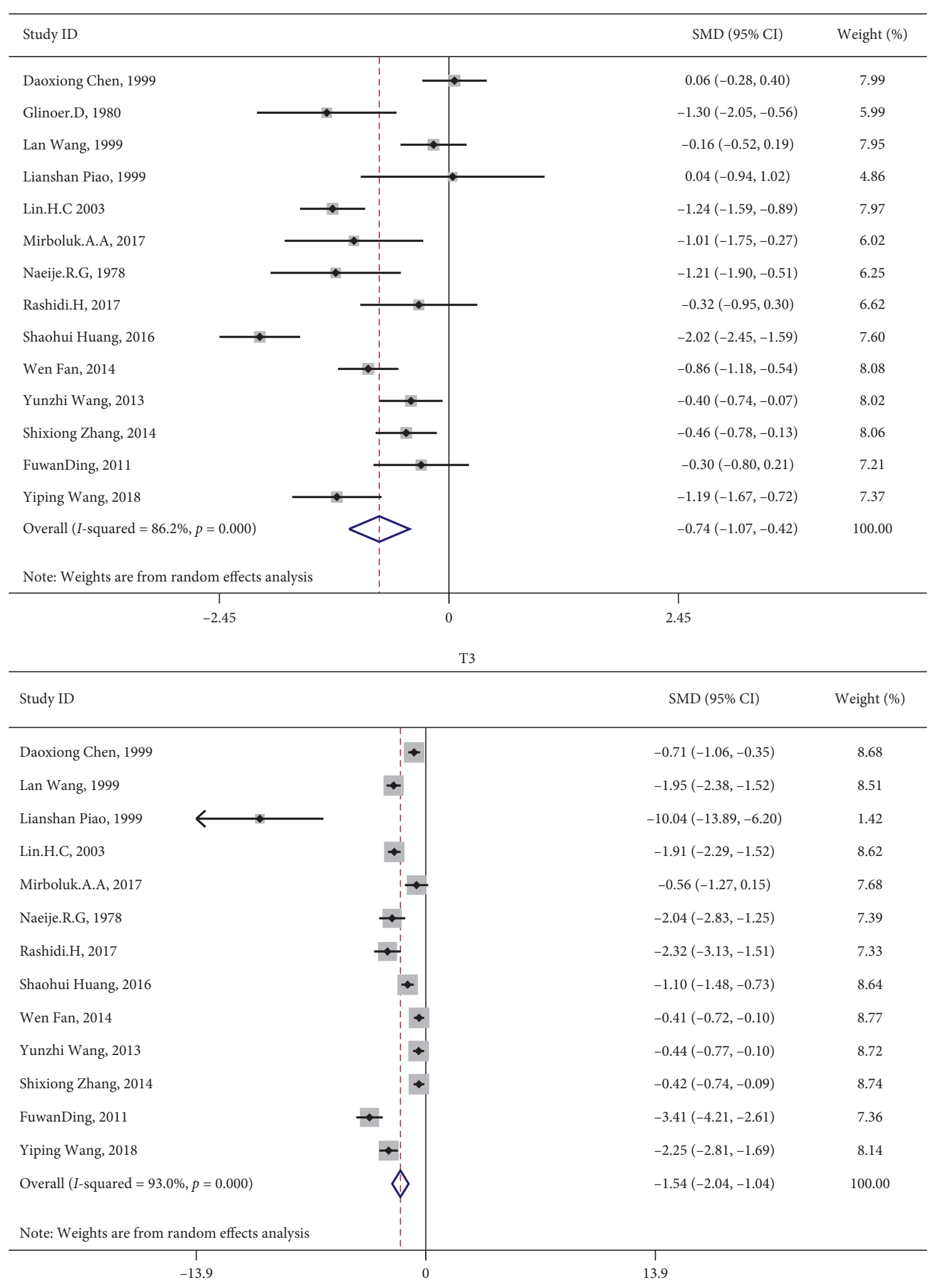

(a)

FIgUre 5: Continued. 
FT4

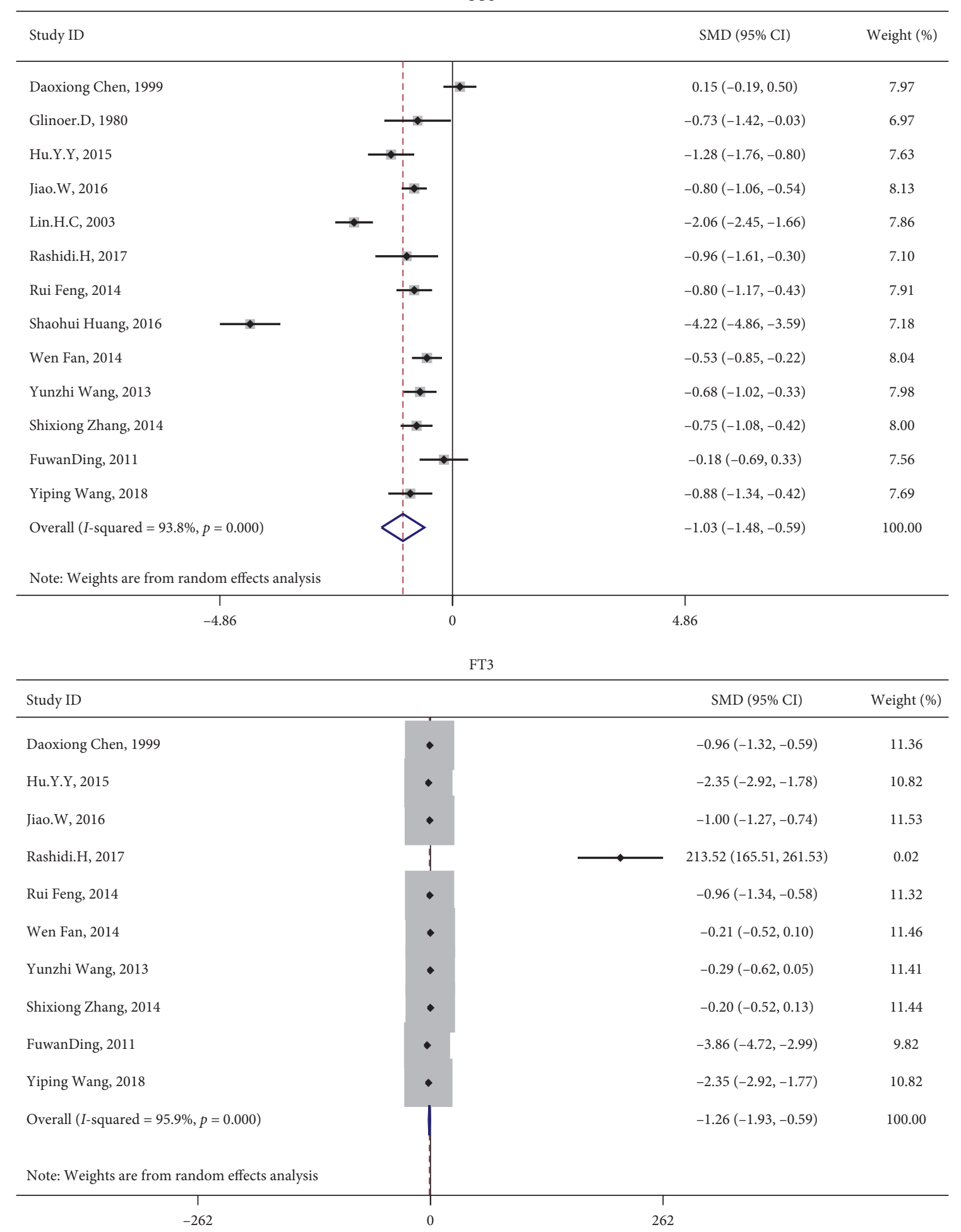

(b)

Figure 5: Continued. 


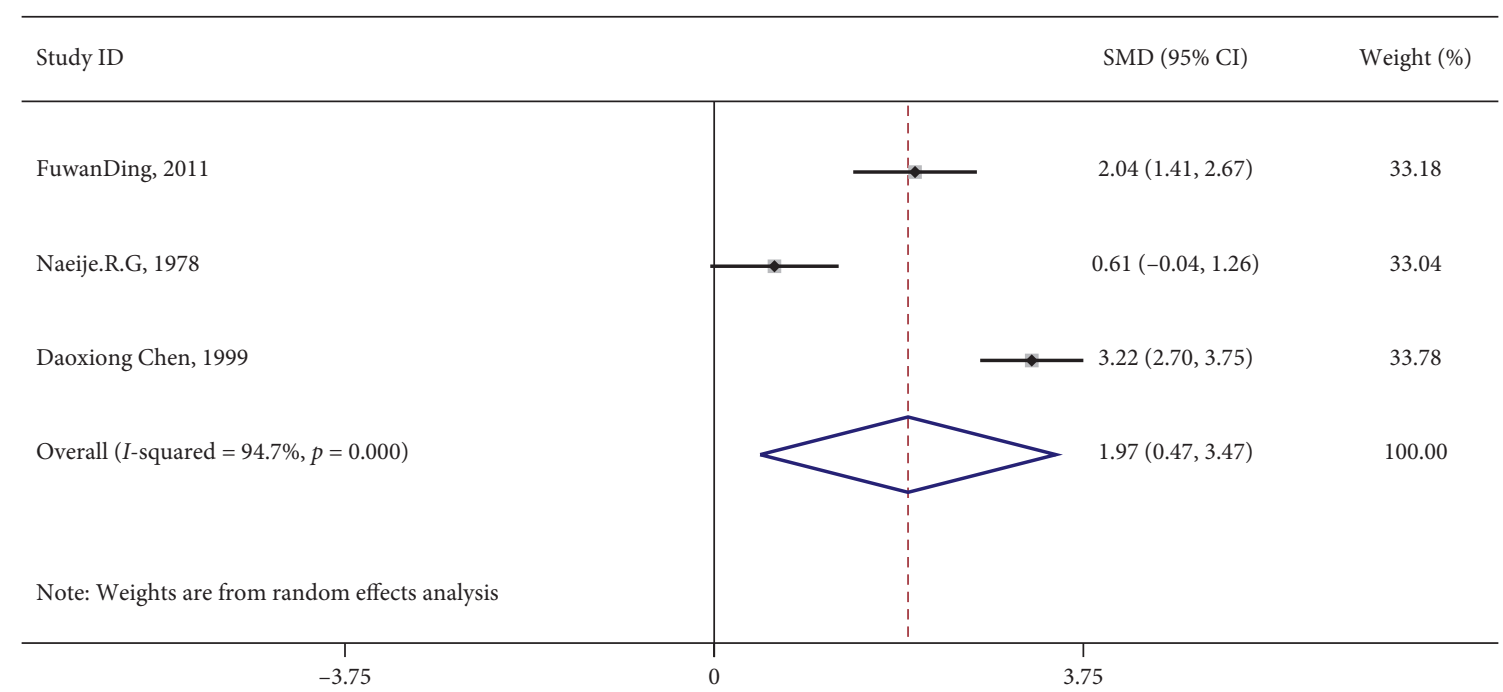

(c)

Figure 5: Forest plot of T4, T3, FT4, FT3, and rT3 compared with patients with DKA before and after treatment.

$\mathrm{T} 4$

Funnel plot with pseudo 95\% confidence limits

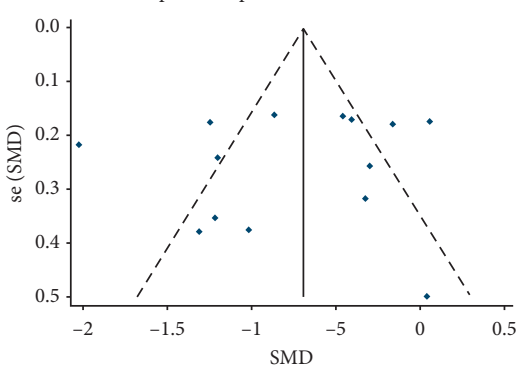

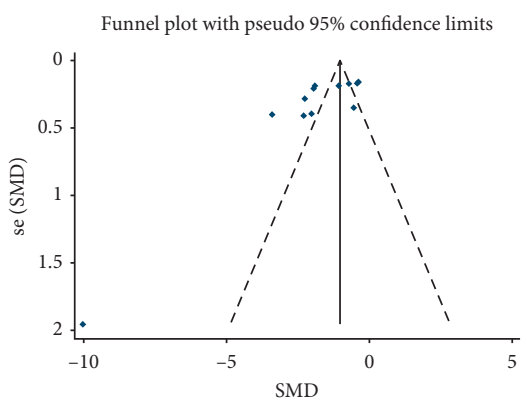

FT3

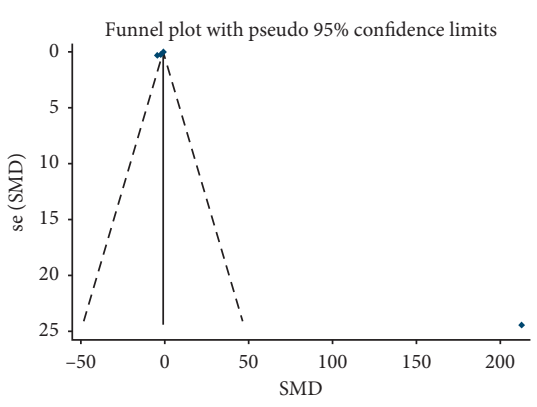

FT4

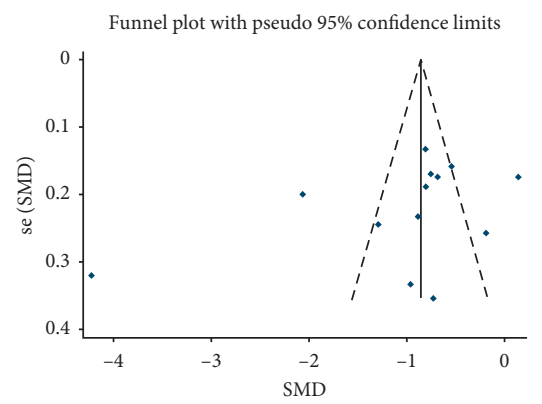

rT3

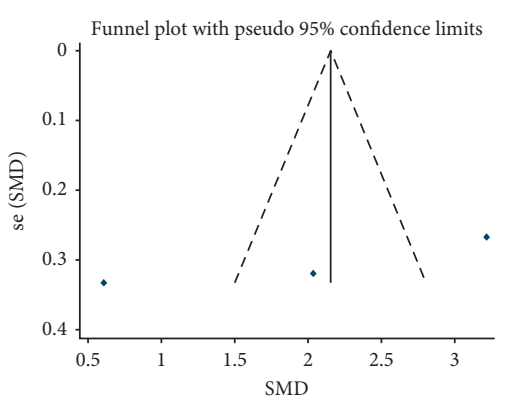

Figure 6: Funnel plot of T4, T3, FT4, FT3, and rT3 compared with patients with DKA before and after treatment.

believed that long-term insulin insufficiency could lead to a more severe and extensive damage of T4 deiodination [55]. Type 2 deiodinase (Dio2) is an intracellular enzyme that catalyzes the conversion of T4 to T3 [62]. A meta-analysis showed that the polymorphism of Dio2 Thr92Ala is associated with poor blood glucose control in patients with T2DM [63].
The limitation of this study is that meta-analysis is a secondary literature analysis based on previous research evidence, so there are limitations and bias in the analysis. The study lacked data for long-term follow-up. The methods used to measure thyroid hormones were much less sensitive than those used in the last decade. 
T4

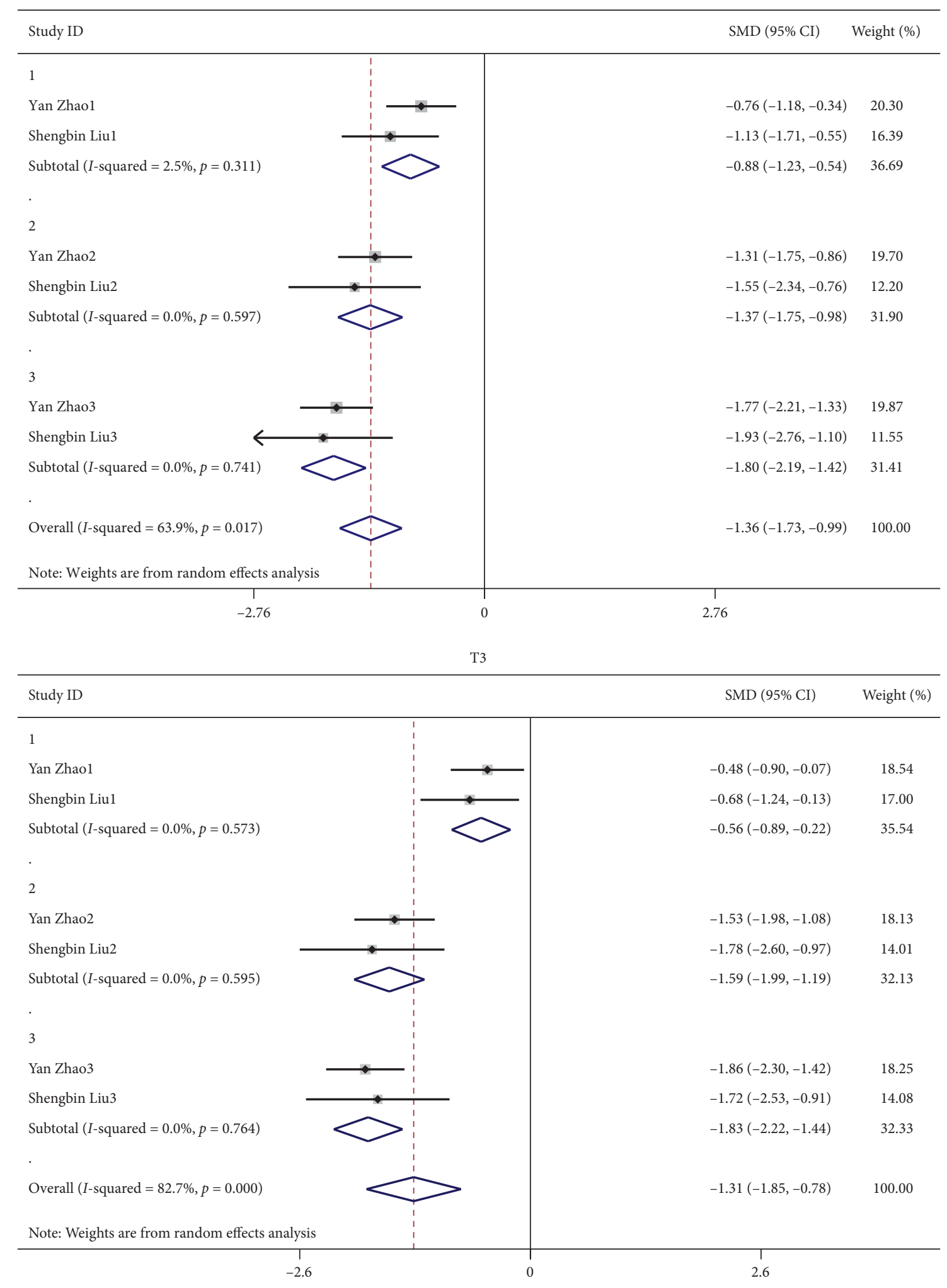

(a)

Figure 7: Continued. 
FT3

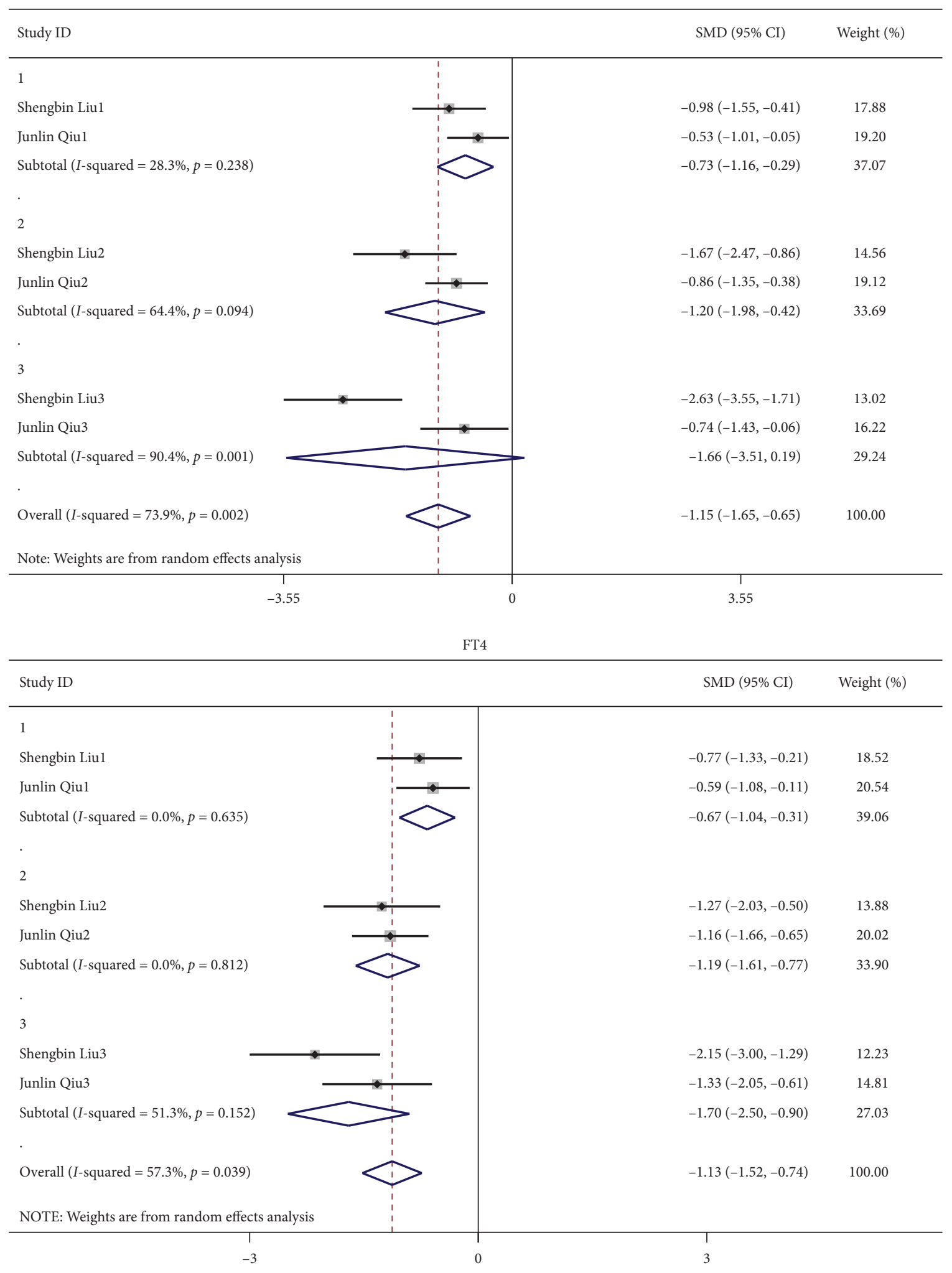

(b)

Figure 7: Comparison of severity of DKA and thyroid function in patients with diabetes and DKA. 


\section{Conclusion}

Thyroid function changed in patients with DKA. It changed with the severity of DKA. This condition may be transient, preceding further recovery of DKA.

\section{Data Availability}

The data used to support the findings of this study are included within the article.

\section{Conflicts of Interest}

The authors declare that they have no conflicts of interest.

\section{References}

[1] E. Karslioglu French, A. C. Donihi, and M. T. Korytkowski, "Diabetic ketoacidosis and hyperosmolar hyperglycemic syndrome: review of acute decompensated diabetes in adult patients," BMJ, vol. 365, p. 11114, 2019.

[2] D. Dabelea, A. Rewers, J. M. Stafford et al., "Trends in the prevalence of ketoacidosis at diabetes diagnosis: the SEARCH for diabetes in youth study," Pediatrics, vol. 133, no. 4, pp. e938-e945, 2014.

[3] O. Pinhas-Hamiel, L. M. Dolan, and P. S. Zeitler, "Diabetic ketoacidosis among obese African-American adolescents with NIDDM," Diabetes Care, vol. 20, no. 4, pp. 484-486, 1997.

[4] C. R. Scott, J. M. Smith, M. M. Cradock, and C. Pihoker, "Characteristics of youth-onset noninsulin-dependent diabetes mellitus and insulin-dependent diabetes mellitus at diagnosis," Pediatrics, vol. 100, no. 1, pp. 84-91, 1997.

[5] A. K. Maniatis, S. H. Goehrig, D. Gao, A. Rewers, P. Walravens, and G. J. Klingensmith, "Increased incidence and severity of diabetic ketoacidosis among uninsured children with newly diagnosed type 1 diabetes mellitus," Pediatric Diabetes, vol. 6, no. 2, pp. 79-83, 2005.

[6] C. M. Alexander, E. M. Kaptein, S. M. C. Lum, C. A. Spencer, D. Kumar, and J. T. Nicoloff, "Pattern of recovery of thyroid hormone indices associated with treatment of diabetes mellitus," The Journal of Clinical Endocrinology \& Metabolism, vol. 54, no. 2, pp. 362-366, 1982.

[7] A. P. Farwell, "Nonthyroidal illness syndrome," Current Opinion in Endocrinology \& Diabetes and Obesity, vol. 20, no. 5, pp. 478-484, 2013.

[8] S. Rai, J. A. Kumar, K. P. Shetty et al., "Thyroid function in type 2 diabetes mellitus and in diabetic nephropathy," Journal of Clinical and Diagnostic Research: JCDR, vol. 7, no. 8, pp. 1583-1585, 2013.

[9] K. K. Dhatariya and G. E. Umpierrez, "Guidelines for management of diabetic ketoacidosis: time to revise?" The Lancet Diabetes \& Endocrinology, vol. 5, no. 5, pp. 321-323, 2017.

[10] D. Chen, Y. Gao, and S. Xu, "Changes of thyroid hormones before and after treatment of diabetic ketoacidosis," Journal of Hainan Medical University, vol. 02, pp. 14-15, 1999.

[11] D. Glinoer, R. Naeije, J. Golstein, M. Fernandez-Deville, and L. Vanhaelst, "Alterations in circulating thyroid hormones and thyroxine-binding globulin levels during diabetic ketoacidosis," Journal of Endocrinological Investigation, vol. 3, no. 1, pp. 67-69, 1980.
[12] L. Wang, "Changes in thyroid function in patients with diabetic ketoacidosis," Zhejiang Medical Journal, vol. 06, pp. 41-42, 1999.

[13] L. Piao and Z. Li, "Changes in insulin antagonist hormones during diabetic ketoacidosis," Journal of Medical Science Yanbian University, vol. 02, pp. 118-120, 1999.

[14] C. H. Lin, Y. J. Lee, C. Y. Huang et al., "Thyroid function in children with newly diagnosed type 1 diabetes mellitus," Acta Paediatrica Taiwanica, vol. 44, no. 3, pp. 145-149, 2003.

[15] A. A. Mirboluk, F. Rohani, R. Asadi, and M. R. Eslamian, "Thyroid function test in diabetic ketoacidosis," Diabetes \& Metabolic Syndrome: Clinical Research \& Reviews, vol. 11, no. Suppl 2, pp. S623-s625, 2017.

[16] R. Naeije, J. Golstein, N. Clumeck, H. Meinhold, K. W. Wenzel, and L. Vanhaelst, "A low T3 syndrome in diabetic ketoacidosis," Clinical Endocrinology, vol. 8, no. 6, 1978.

[17] H. Rashidi, S. B. Ghaderian, S. M. Latifi, and F. Hoseini, "Impact of diabetic ketoacidosis on thyroid function tests in type 1 diabetes mellitus patients," Diabetes \& Metabolic Syndrome: Clinical Research \& Reviews, vol. 11, no. Suppl 1, pp. S57-S59, 2017.

[18] S. Huang and L. Su, "Detection and analysis of TT3, FT4, and TT4 levels in patients with diabetic ketoacidosis," Shenzhen Journal of Integrated Traditional Chinese and Western Medicine, vol. 03, pp. 64-65, 2016.

[19] W. Fan, "Changes of thyroid hormones levels in elderly diabetic ketoacidosis patients," Journal of Clinical and Experimental Medicine, vol. 06, pp. 481-483, 2014.

[20] Y. Wang and J. Du, "Study on serum thyroid hormones levels in elderly patients with diabetes and ketoacidosis," Chinese General Practice, vol. 10, pp. 876-877, 2013.

[21] S. Zhang, "Clinical analysis of thyroid hormones in elderly diabetic ketoacidosis patients," For All Health, vol. 8, no. 03, p. 116, 2014.

[22] F. Ding and M. Ji, "Clinical analysis of 30 cases of diabetic ketoacidosis with low T3 syndrome," Chinese Journal of Clinical Research, vol. 24, no. 12, pp. 1101-1102, 2011.

[23] Y. Wang, F. Song, and G. Li, "Study on serum thyroid hormone levels in children with type 1 diabetes and ketoacidosis," Journal of North Sichuan Medical College, vol. 33, no. 03, pp. 450-453, 2018.

[24] Y.-Y. Hu, G.-M. Li, and W. Wang, "Euthyroid sick syndrome in children with diabetic ketoacidosis," Saudi Medical Journal, vol. 36, no. 2, pp. 243-247, 2015.

[25] W.-J. Jiao, H. Li, T.-Y. Li, T. Feng, and S.-J. Li, "Effect of insulin pump infusion on comprehensive stress state of patients with diabetic ketoacidosis," Tropical Journal of Pharmaceutical Research, vol. 15, no. 10, pp. 2283-2287, 2016.

[26] R. Feng, "Changes in general stress status of patients with diabetic ketoacidosis before and after insulin pump treatment," Journal of Hainan Medical University, vol. 10, pp. 1365-1370, 2014.

[27] C. M. Alexander, S. M. Lum, J. Rhodes, C. Boarman, J. T. Nicoloff, and D. Kumar, "Rapid increase in both plasma fibronectin and serum triiodothyromine associated with treatment of diabetic ketoacidosis," The Journal of Clinical Endocrinology and Metabolism, vol. 56, no. 2, pp. 279-282, 1983.

[28] F. Chiarelli, S. Tumini, A. Verrotti, and G. Morgese, "Effects of ketoacidosis and puberty on basal and TRH-stimulated thyroid hormones and TSH in children with diabetes 
mellitus," Hormone and Metabolic Research, vol. 21, no. 09, pp. 494-497, 1989.

[29] L. Luo, D. Deng, and C. Wang, "Changes of thyroid hormone levels in patients with diabetic ketoacidosis," Anhui Medical and Pharmaceutical Journal, vol. 09, pp. 1317-1318, 2012.

[30] J. L. Schlienger, A. Anceau, G. Chabrier, M. L. North, and F. Stephan, "Effect of diabetic control on the level of circulating thyroid hormones," Diabetologia, vol. 22, no. 6, pp. 486-488, 1982.

[31] Y. Zhao, B. Ynag, L. Huang et al., "201 cases of type 1 diabetes with low triiodothyronine syndrome," Chinese Journal of Applied Clinical Pediatrics, vol. 08, pp. 594-610, 2012.

[32] Y. Qiao, "Analysis of thyroid function in patients with diabetic ketoacidosis," Master thesis, Beijing University of Chinese Medicine, Beijing, China, 2012.

[33] S. Liu, "To explore the relationship between the changes of thyroid function and the condition in patients with diabetic ketoacidosis and hyperglycemia hypertonicity," Master thesis, Guangxi Medical University, Nanning, China, 2016.

[34] Q. Chen, M. Lu, Z. Ji et al., "Analysis of serum thyroid hormone levels in diabetic patients with ketoacidosis," Preventive Medicine, vol. 28, no. 03, pp. 268-269+273, 2016.

[35] B. Liu, "Study on the relationship between serum thyroid stimulating hormone and thyroid hormone levels in patients with type 2 diabetes," Proceeding of Clinical Medicine, vol. 04, pp. 284-286, 2012.

[36] J. Qiu, H. Qiu, H. Wang et al., "Effect of diabetic ketoacidosis on thyroid function," Chinese Journal of Diabetes, vol. 09, pp. 756-759.37, 2018.

[37] Y. Xin, M. Yang, X. J. Chen, Y. J. Tong, and L. H. Zhang, "Clinical features at the onset of childhood type 1 diabetes mellitus in Shenyang, China," Journal of Paediatrics and Child Health, vol. 46, no. 4, pp. 171-175, 2010.

[38] K. Derkach, I. Bogush, L. Berstein, and A. Shpakov, "The influence of intranasal insulin on hypothalamic-pituitarythyroid Axis in normal and diabetic rats," Hormone and Metabolic Research, vol. 47, no. 12, pp. 916-924, 2015.

[39] L. Piconi, L. Quagliaro, R. Da Ros et al., "Intermittent high glucose enhances ICAM-1, VCAM-1, E-selectin and interleukin-6 expression in human umbilical endothelial cells in culture: the role of poly(ADP-ribose) polymerase," Journal of Thrombosis and Haemostasis, vol. 2, no. 8, pp. 1453-1459, 2004.

[40] S. M. Adler and L. Wartofsky, "The nonthyroidal illness syndrome," Endocrinology and Metabolism Clinics of North America, vol. 36, no. 3, pp. 657-672, 2007.

[41] A. Boelen, M. C. Platvoet-Ter Schiphorst, and W. M. Wiersinga, "Association between serum interleukin-6 and serum 3,5,3'-triiodothyronine in nonthyroidal illness," The Journal of Clinical Endocrinology \& Metabolism, vol. 77, no. 6, pp. 1695-1699, 1993.

[42] J. Köhrle, "Thyroid hormone transporters in health and disease: advances in thyroid hormone deiodination," Best Practice \& Research Clinical Endocrinology \& Metabolism, vol. 21, no. 2, pp. 173-191, 2007.

[43] Y. Tomer and F. Menconi, "Type 1 diabetes and autoimmune thyroiditis: the genetic connection," Thyroid, vol. 19, no. 2, pp. 99-102, 2009.

[44] I. Elebrashy, A. El Meligi, L. Rashed, R. F. Salam, E. Youseef, and S. A. Fathy, "Thyroid dysfunction among type 2 diabetic female Egyptian subjects," Therapeutics and Clinical Risk Management, vol. 12, pp. 1757-1762, 2016.

[45] C. Balsamo, S. Zucchini, G. Maltoni et al., "Relationships between thyroid function and autoimmunity with metabolic derangement at the onset of type 1 diabetes: a cross-sectional and longitudinal study," Journal of Endocrinological Investigation, vol. 38, no. 6, pp. 701-707, 2015.

[46] S. H. Song, I. S. Kwak, D. W. Lee, Y. H. Kang, E. Y. Seong, and J. S. Park, "The prevalence of low triiodothyronine according to the stage of chronic kidney disease in subjects with a normal thyroid-stimulating hormone," Nephrology Dialysis Transplantation, vol. 24, no. 5, pp. 1534-1538, 2009.

[47] B. Malekpour, A. Mehrafshan, F. Saki, Z. Malekmohammadi, and N. Saki, "Effect of posttraumatic serum thyroid hormone levels on severity and mortality of patients with severe traumatic brain injury," Acta Medica Iranica, vol. 50, no. 2, pp. 113-116, 2012.

[48] S. Bernasconi, M. Vanelli, G. Nori et al., "Serum TSH, T4, T3, FT4, FT3, rT3, and TBG in youngsters with non-ketotic insulin-dependent diabetes mellitus," Hormone Research, vol. 20, no. 4, pp. 213-217, 1984.

[49] H. Dorchy, P. Bourdoux, and B. Lemiere, "Subclinical thyroid hormone abnormalities in type I diabetic children and adolescents. Relationship to metabolic control," Acta Paediatrica, vol. 74, no. 3, pp. 386-389, 1985.

[50] S. Salardi, A. Fava, A. Cassio et al., "Thyroid function and prolactin levels in insulin-dependent diabetic children and adolescents," Diabetes, vol. 33, no. 6, pp. 522-526, 1984.

[51] S. Madsbad, P. Laurberg, J. Weeke et al., "Very early changes in circulating T3 and rT3 during development of metabolic derangement in diabetic patients," Acta Medica Scandinavica, vol. 209, no. 5, pp. 385-387, 1981.

[52] M. H. Warner and G. J. Beckett, "Mechanisms behind the non-thyroidal illness syndrome: an update," Journal of Endocrinology, vol. 205, no. 1, pp. 1-13, 2010.

[53] A. Burger, P. Suter, P. Nicod, M. B. Vallotton, A. Vagenakis, and L. Braverman, "Reduced active thyroid hormone levels in acute illness," The Lancet, vol. 307, no. 7961, pp. 653-655, 1976.

[54] M. Schimmel and R. D. Utiger, "Thyroidal and peripheral production of thyroid hormones," Annals of Internal Medicine, vol. 87, no. 6, pp. 760-768, 1977.

[55] C. S. Pittman, A. K. Suda, J. B. Chambers Jr., H. G. McDaniel, G. Y. Ray, and B. K. Preston, "Abnormalities of thyroid hormone turnover in patients with diabetes mellitus before and after insulin therapy," The Journal of Clinical Endocrinology \& Metabolism, vol. 48, no. 5, pp. 854-860, 1979.

[56] M. Inada, J. Okabe, Y. Kazama, H. Takayama, T. Nakagawa, and K. Torizuka, "Thyroxine turnover and transport in diabetes mellitus," The Journal of Clinical Endocrinology \& Metabolism, vol. 36, no. 3, pp. 590-597, 1973.

[57] S. W. Spaulding, I. J. Chopra, R. S. Sherwin, and S. S. Lyall, "Effect of caloric restriction and dietary composition on serum T3and reverse T3in man," The Journal of Clinical Endocrinology \& Metabolism, vol. 42, no. 1, pp. 197-200, 1976.

[58] G. Bray, D. Fisher, and I. Chopra, "Relation of thyroid hormones to body-weight," The Lancet, vol. 307, no. 7971, pp. 1206-1208, 1976.

[59] A. R. C. Harris, S.-L. Fang, A. G. Vagenakis, and L. E. Bravernan, "Effect of starvation, nutriment replacement, and hypothyroidism on in vitro hepatic T4 to T3 conversion in the rat," Metabolism, vol. 27, no. 11, pp. 1680-1690, 1978.

[60] S. L. Welle and R. G. Campbell, "Decrease in resting metabolic rate during rapid weight loss is reversed by low dose thyroid hormone treatment," Metabolism, vol. 35, no. 4, pp. 289-291, 1986. 
[61] C. S. Pittman, A. K. Suda, J. B. Chambers Jr., and G. Y. Ray, "Impaired 3,5,3' -triiodothyronine (T3) production in diabetic patients," Metabolism, vol. 28, no. 4, pp. 333-338, 1979.

[62] A. C. Bianco, D. Salvatore, B. Gereben, M. J. Berry, and P. R. Larsen, "Biochemistry, cellular and molecular biology, and physiological roles of the iodothyronine selenodeiodinases," Endocrine Reviews, vol. 23, no. 1, pp. 38-89, 2002.

[63] X. Zhang, J. Sun, W. Han et al., "The type 2 deiodinase Thr92Ala polymorphism is associated with worse glycemic control in patients with type 2 diabetes mellitus: a systematic review and meta-analysis," Journal of Diabetes Research, vol. 2016, Article ID 5928726, , 2016. 